\title{
ERROR ESTIMATES OF SEMI-DISCRETE AND FULLY DISCRETE FINITE ELEMENT METHODS FOR THE CAHN-HILLIARD-COOK EQUATION *
}

\author{
RUISHENG $\mathrm{QI}^{\dagger}$ AND XIAOJIE WANG W $^{\dagger}$
}

\begin{abstract}
In two recent publications [Kovács, Larsson, and Mesforush, SIAM J. Numer. Anal. 49(6), 2407-2429, 2011] and [Furihata, et al., SIAM J. Numer. Anal. 56(2), 708-731, 2018], strong convergence of the semi-discrete and fully discrete finite element methods is, respectively, proved for the Cahn-Hilliard-Cook (CHC) equation, but without convergence rates revealed. The present work aims to fill the left gap, by recovering strong convergence rates of (fully discrete) finite element methods for the CHC equation. More accurately, strong convergence rates of a full discretization are obtained, based on Galerkin finite element methods for the spatial discretization and the backward Euler method for the temporal discretization. It turns out that the convergence rates heavily depend on the spatial regularity of the noise process. Different from the stochastic Allen-Cahn equation, the presence of the unbounded elliptic operator in front of the cubic nonlinearity in the underlying model makes the error analysis much more challenging and demanding. To address such difficulties, several new techniques and error estimates are developed. Numerical examples are finally provided to confirm the previous findings.
\end{abstract} rates

Key words. Cahn-Hilliard-Cook equation, finite element method, backward Euler method, strong convergence

AMS subject classifications. 60H35, 60H15, 65C30

1. Introduction. Over the last twenty years, numerical approximations of stochastic partial differential equations (SPDEs) with globally Lipschitz coefficients have been extensively and well studied, see the monographs [24, 29, 35] and references therein. By contrast, numerical analysis of SPDEs with non-globally Lipschitz coefficients is, in our opinion, at an early stage and far from being well-understood. A typical SPDE model with non-globally Lipschitz coefficients is the stochastic Allen-Cahn equation, which has been numerically studied by many researchers recently, see, e.g., [1 5, 9, 11, 13, 17, 18, 20, 22, 23, 25, 26, 33, 34, 36. As another prominent SPDE model with non-globally Lipschitz coefficients, the Cahn-Hilliard-Cook (CHC) equations, also known as the stochastic CahnHilliard equation in some literature, are, however, much less investigated. As far as we know, only a few publications are devoted to numerical studies of the CHC equation 10,12,19, 21, 27, 32. Particularly, strong convergence of the semi-discrete and fully discrete finite element methods is, respectively, proved in 27] and [19] for the CHC equation, but without convergence rates recovered. The present article attempts to fill the left gap, by recovering strong convergence rates of the (fully discrete) finite element methods for the CHC equation.

Let $D \subset \mathbb{R}^{d}, d \in\{1,2,3\}$ be a bounded convex spatial domain with smooth boundary and let $H:=L_{2}(D ; \mathbb{R})$ be the real separable Hilbert space endowed with the usual inner product and norm. Throughout the paper we are interested in the following Cahn-Hilliard-Cook equation perturbed by

* R.Q. was supported by National Science Foundation of China (11701073). X.W. was supported by National Science Foundation of China $(11671405,11971488,91630312)$ and Natural Science Foundation of Hunan Province for Distinguished Young Scholars (2020JJ2040). Dr. Meng Cai is gratefully acknowledged for bringing a few typos into our notice. Also, the authors want to thank the Tianyuan Mathematical Center in Northeast China for the hospitality and Prof. Xiaobing Feng from University of Tennessee for his helpful comments when this work was first presented in a conference in June of 2018, hosted by the center.

${ }^{\dagger}$ School of Mathematics and Statistics, Northeastern University at Qinhuangdao, Qinhuangdao, China (qirsh@neuq.edu.cn).

${ }^{\ddagger}$ School of Mathematics and Statistics, Central South University, Changsha 410083, Hunan, China (x.j.wang7@csu.edu.cn, $x \cdot j \cdot$ wang7@gmail.com). 
noise in $\dot{H}:=\left\{v \in H: \int_{D} v d x=0\right\}$,

$$
\begin{cases}\mathrm{d} u-\Delta w \mathrm{~d} t=\mathrm{d} W, & \text { in } D \times(0, T], \\ w=-\Delta u+f(u), & \text { in } D \times(0, T], \\ \frac{\partial u}{\partial n}=\frac{\partial w}{\partial n}=0, & \text { in } \partial D \times(0, T], \\ u(0, x)=u_{0}, & \text { in } D,\end{cases}
$$

where $\Delta=\sum_{i=1}^{d} \frac{\partial^{2}}{\partial x_{i}^{2}}, f(s)=s^{3}-s, s \in \mathbb{R}$ and $\frac{\partial}{\partial n}$ denotes the outward normal derivative on $\partial D$. Following the framework of [14] we rewrite (1.1) as an abstract evolution equation of the form,

$$
\left\{\begin{array}{l}
\mathrm{d} X(t)+A(A X(t)+F(X(t))) \mathrm{d} t=\mathrm{d} W(t), \quad t \in(0, T], \\
X(0)=X_{0}
\end{array}\right.
$$

where $-A$ is the Laplacian with homogeneous Neumann boundary conditions and $-A^{2}$ generates an analytic semigroup $E(t)$ in $\dot{H}$. Similarly as in [19,27, $\{W(t)\}_{t \geq 0}$ is assumed to be an $\dot{H}$-valued $Q$-Wiener process on a filtered probability space $\left(\Omega, \mathcal{F}, \mathbb{P},\left\{\mathcal{F}_{t}\right\}_{t \geq 0}\right)$. The nonlinear mapping $F$ is supposed to be a Nemytskij operator, given by $F(u)(x)=f(u(x)), x \in D$.

The deterministic version of such equation is used to describe the complicated phase separation and coarsening phenomena in a melted alloy [6] that is quenched to a temperature at which only two different concentration phases can exist stably. For such model, $u$ represents the concentration of an alloy and $w$ models the chemical potential. The corresponding numerical study, e.g., can be consulted in [16]. Concerning the stochastic version, Da Prato and Debussche [14] have already proved the existence and uniqueness of the solution to (1.2). The space-time regularities of the weak solution of (1.2) have been further examined in [19,30. As the first goal, this work aim to provide improved regularity results for the solution to (1.2) based on existing ones from [14, 19, 30. Under further assumptions specified later, particularly including

$$
\left\|A^{\frac{\gamma-2}{2}} Q^{\frac{1}{2}}\right\|_{\mathcal{L}_{2}}<\infty, \text { for some } \gamma \in[3,4],
$$

Theorems 2.1, 2.2 assert that, (1.2) admits a unique mild solution $X(t), t \in[0, T]$, given by

$$
X(t)=E(t) X_{0}-\int_{0}^{t} E(t-s) A F(X(s)) \mathrm{d} s+\int_{0}^{t} E(t-s) \mathrm{d} W(s)
$$

which enjoys the following spatial-temporal regularity properties,

$$
X \in L_{\infty}\left([0, T] ; L^{p}\left(\Omega ; \dot{H}^{\gamma}\right)\right), \forall p \geq 1,
$$

and for $\forall p \geq 1$ and $0 \leq s<t \leq T$,

$$
\|X(t)-X(s)\|_{L^{p}\left(\Omega, \dot{H}^{\beta}\right)} \leq C(t-s)^{\min \left\{\frac{1}{2}, \frac{\gamma-\beta}{4}\right\}}, \beta \in[0, \gamma] .
$$

Here $\dot{H}^{\alpha}:=\operatorname{dom}\left(A^{\frac{\alpha}{2}}\right), \alpha \in \mathbb{R}$ and the parameter $\gamma \in[3,4]$ coming from (1.3) quantifies the spatial regularity of the covariance operator $Q$ of the driving noise process.

The second aim of this article is to derive error estimates for finite element approximations of the stochastic problem (1.2). By $\dot{V}_{h} \subset H^{1}(D) \cap \dot{H}$ we denote the space of continuous functions that are piecewise polynomials of degree at most $r-1$, for $r \in\{2,3,4\}$ in dimension $d=1$ and $r=2$ in dimension $d \in\{2,3\}$, and by $X_{h}(t) \in \dot{V}_{h}$ the finite element spatial approximation of the mild solution $X$, represented by

$$
X_{h}(t)=E_{h}(t) P_{h} X_{0}-\int_{0}^{t} E_{h}(t-s) A_{h} P_{h} F\left(X_{h}(s)\right) \mathrm{d} s+\int_{0}^{t} E_{h}(t-s) P_{h} \mathrm{~d} W(s), \quad t \in[0, T] .
$$


Here $h>0$ is the mesh size and $E_{h}(t):=e^{-t A_{h}^{2}}$ is the strongly continuous semigroup generated by the discrete operator $-A_{h}^{2}$. The resulting spatial approximation error, as implied by Theorem 4.2 is measured as follows,

$$
\left\|X(t)-X_{h}(t)\right\|_{L^{p}(\Omega ; \dot{H})} \leq C h^{\kappa}|\ln h|, \quad \kappa:=\min \{\gamma, r\} .
$$

To arrive at it, we introduce an auxiliary approximation process $\widetilde{X}_{h}$, defined by

$$
\tilde{X}_{h}(t)=E_{h}(t) P_{h} X_{0}-\int_{0}^{t} E_{h}(t-s) A_{h} P_{h} F(X(s)) \mathrm{d} s+\int_{0}^{t} E_{h}(t-s) P_{h} \mathrm{~d} W(s), \quad t \in[0, T],
$$

and split the considered error $\left\|X(t)-X_{h}(t)\right\|_{L^{p}(\Omega ; \dot{H})}$ into two parts:

$$
\left\|X(t)-X_{h}(t)\right\|_{L^{p}(\Omega ; \dot{H})} \leq\left\|X(t)-\widetilde{X}_{h}(t)\right\|_{L^{p}(\Omega ; \dot{H})}+\left\|\widetilde{X}_{h}(t)-X_{h}(t)\right\|_{L^{p}(\Omega ; \dot{H})} .
$$

In a semigroup framework, one can straightforwardly treat the first error term and show $\| X(t)-$ $\widetilde{X}_{h}(t) \|_{L^{p}(\Omega ; \dot{H})}=O\left(h^{\kappa}|\ln h|\right)$, with the aid of the well-known estimates for the error operators $\Psi_{h}(t):=$ $E(t)-E_{h}(t) P_{h}$ and $\Phi_{h}(t):=E(t) A-E_{h}(t) A_{h} P_{h}$ and uniform moment bounds of $\widetilde{X}_{h}(t)$ and $X(t)$. Further, we subtract (1.5) from (1.7) to eliminate the stochastic convolution and the remaining term $\widetilde{e}(t):=\widetilde{X}_{h}(t)-X_{h}(t)$ satisfies

$$
\mathrm{d} \widetilde{e}_{h}(t)+A_{h}^{2} \widetilde{e}_{h}(t) \mathrm{d} t=A_{h} P_{h}\left(F\left(X_{h}(t)\right)-F(X(t))\right) \mathrm{d} t, \quad \widetilde{e}_{h}(0)=0,
$$

whose solution is given by

$$
\widetilde{e}_{h}(t)=\int_{0}^{t} E_{h}(t-s) A_{h} P_{h}\left(F\left(X_{h}(s)\right)-F(X(s))\right) \mathrm{d} s .
$$

Note that the tough term $\|\widetilde{e}(t)\|_{L^{p}(\Omega ; \dot{H})}$ can not be handled directly due to the presence of $A_{h}$ before the nonlinearity. However, we turn things around and derive $\left\|\int_{0}^{t}\left|\widetilde{e}_{h}(s)\right|_{1}^{2} \mathrm{~d} s\right\|_{L^{p}(\Omega ; \mathbb{R})}=O\left(h^{2 \kappa}|\ln h|^{2}\right)$ instead, after fully exploiting (1.9), the monotonicity of the nonlinearity, regularity properties of $X_{h}(t)$, $\widetilde{X}_{h}(t)$ and $X(t)$, and the previous error estimate for $\left\|X(t)-\widetilde{X}_{h}(t)\right\|_{L^{p}(\Omega ; \dot{H})}$. Equipped with the key error estimate of $\left\|\int_{0}^{t}\left|\widetilde{e}_{h}(s)\right|_{1}^{2} \mathrm{~d} s\right\|_{L^{p}(\Omega ; \mathbb{R})}$ and (1.10), we can smoothly show $\left\|\widetilde{X}_{h}(t)-X_{h}(t)\right\|_{L^{p}(\Omega ; \dot{H})}=$ $O\left(h^{\kappa}|\ln h|\right)($ see (4.20)-(4.27) ) and therefore obtain (1.6).

Let $k=T / N, N \in \mathbb{N}$ be a uniform time step-size. After discretizing the stochastic problem (1.2) by the finite element method in space and the backward Euler scheme in time, we also investigate the resulting fully discrete scheme, given by

$$
X_{h}^{n}=E_{k, h} X_{h}^{n-1}-k E_{k, h} A_{h} P_{h} F\left(X_{h}^{n}\right)+E_{k, h} P_{h} \Delta W_{n},
$$

where $E_{k, h}:=\left(I+k A_{h}^{2}\right)^{-1}$ and $X_{h}^{n}$ is regarded as the fully discrete approximation of $X\left(t_{n}\right)$. By essentially exploiting discrete analogue of arguments as used in the semi-discrete case, one can obtain the following strong approximation error bound

$$
\left\|X\left(t_{n}\right)-X_{h}^{n}\right\|_{L^{p}(\Omega ; \dot{H})} \leq C\left(h^{\kappa}|\ln h|+k^{\frac{\kappa}{4}}|\ln k|\right), \quad \kappa:=\min \{\gamma, r\} .
$$

It is important to mention that, the presence of the unbounded operator $A$ in front of the nonglobally Lipschitz (cubic) nonlinearity in the underlying model causes essential difficulties in the error analysis for the approximations and the error analysis becomes much more challenging than that of the stochastic Allen-Cahn equation (see [37] and relevant comments in [19,27]). More specifically, our 
error analysis heavily relies on the new approach mentioned before, a priori strong moment bounds of the numerical approximations, and a variety of error estimates for the finite element approximation of the corresponding deterministic linear problem. Some estimates can be derived from existing ones in [19, 28, 30]. Nevertheless, estimates available in [19,28, 30] are far from being enough for the purpose of the error analysis. For example, the strong moment bounds (3.20) and (5.2) and the error estimates of integral form such as (4.6), (4.7), (6.6) and (6.7) are completely new.

Finally, we add some comments on a few closely relevant works. A finite difference scheme was examined in [10] for the problem (1.2) and convergence in probability was established with rates. Hutzenthaler and Jentzen [21] used a general perturbation theory and exponential integrability properties of the exact and numerical solutions to prove strong convergence rates for the spatial spectral Galerkin approximation (no time discretizaton) in one spatial dimension. In [19, 30, strong convergence of finite element methods for (1.2) was proved, but with no rate obtained. The analysis in [19.30] is based on proving a priori moment bounds with large exponents and in higher order norms using energy arguments and bootstrapping followed by a pathwise Gronwall argument in the mild solution setting. Before submitting the early version of the present work to arXiv in late December of 2018, we were also aware of an interesting preprint [12] submitted to arXiv in mid-December of 2018. There strong convergence rates of a fully discrete scheme are obtained, done by a spatial spectral Galerkin method and a temporal accelerated implicit Euler method for the CHC equations. To the best of our knowledge, strong convergence rates of finite element methods for the CHC equations are missing in the existing literature and this article fills the left gap.

The outline of this paper is as follows. In the next section, some preliminaries are collected and certain assumptions are made to ensure well-posedness of the considered problem. Section 3 is devoted to the uniform moment bounds of the semi-discrete finite element approximation. Based on the uniform moment bounds obtained in section 3, we derive the error estimates for the semi-discrete problem in section 4. Section 5 focuses on the uniform moment bounds of the fully discrete approximations and section 6 provides error estimates of the backward Euler-finite element full discretization. In section 7 numerical examples are provided to confirm the previous findings.

2. The Cahn-Hilliard-Cook equation. Throughout this paper, we use $\mathbb{N}$ to denote the set of all positive integers and denote $\mathbb{N}_{0}=\{0\} \cup \mathbb{N}$. Given a separable $\mathbb{R}$-Hilbert space $(H,(\cdot, \cdot)$, $\|\cdot\|)$, by $\mathcal{L}(H)$ we denote the Banach space of all linear bounded operators from $H$ to $H$. Also, we denote by $\mathcal{L}_{2}(H)$ the Hilbert space consisting of all Hilbert-Schmidt operators from $H$ into $H$, equipped with the inner product and the norm,

$$
\left\langle\Gamma_{1}, \Gamma_{2}\right\rangle_{\mathcal{L}_{2}(H)}=\sum_{j=1}^{\infty}\left\langle\Gamma_{1} \phi_{j}, \Gamma_{2} \phi_{j}\right\rangle, \quad\|\Gamma\|_{\mathcal{L}_{2}(H)}=\left(\sum_{j=1}^{\infty}\left\|\Gamma \phi_{j}\right\|^{2}\right)^{\frac{1}{2}}
$$

independent of the choice of orthonormal basis $\left\{\phi_{j}\right\}$ of $H$. If $\Gamma \in \mathcal{L}_{2}(H)$ and $L \in \mathcal{L}(H)$, then $\Gamma L, L \Gamma \in \mathcal{L}_{2}(H)$ and

$$
\|\Gamma L\|_{\mathcal{L}_{2}(H)} \leq\|L\|_{\mathcal{L}(H)}\|\Gamma\|_{\mathcal{L}_{2}(H)}, \quad\|L \Gamma\|_{\mathcal{L}_{2}(H)} \leq\|L\|_{\mathcal{L}(H)}\|\Gamma\|_{\mathcal{L}_{2}(H)}
$$

2.1. Abstract framework and main assumptions. In this subsection, we formulate main assumptions concerning the operator $A$, the nonlinear mapping, the noise process and the initial data.

Assumption 2.1. (Linear operator $A$ ) Let $D$ be a bounded convex domain in $\mathbb{R}^{d}$ for $d \in\{1,2,3\}$ with a sufficiently smooth boundary $\partial D$ and let $H=L_{2}(D ; \mathbb{R})$ be the real separable Hilbert space endowed with the usual inner product $(\cdot, \cdot)$ and the associated norm $\|\cdot\|=(\cdot, \cdot)^{\frac{1}{2}}$. Let $\dot{H}=\{v \in H$ : $\left.(v, 1)=\int_{D} v \mathrm{~d} x=0\right\}$ and let $-A=\Delta$ be the Neumann Laplacian, with the domain $\operatorname{dom}(A):=\{v \in$ $H^{2}(D) \cap \dot{H}: \frac{\partial v}{\partial n}=0$, on $\left.\partial D\right\}$. 
Such assumptions guarantee that the operator $A$ is positive definite, self-adjoint, unbounded, linear on $\dot{H}$ with compact inverse. Let $P: H \rightarrow \dot{H}$ denote a generalized orthogonal projection, given by $P v=v-|D|^{-1} \int_{D} v \mathrm{~d} x$. Then $(I-P) v=|D|^{-1} \int_{D} v \mathrm{~d} x$ is the average of $v$ and it is not difficult to check

$$
\|P v\|_{L_{q}} \leq 2\|v\|_{L_{q}}, \quad q \geq 2 .
$$

Here and below, by $L_{r}(D ; \mathbb{R}), r \geq 1\left(L_{r}(D)\right.$ or $L_{r}$ for short) we denote a Banach space consisting of $r$-times integrable functions. When extended to $H$ as $A v:=A P v$, for $v \in H$, the linear operator $A$ has an orthonormal basis $\left\{e_{j}\right\}_{j \in \mathbb{N}_{0}}$ of $H$ with corresponding eigenvalues $\left\{\lambda_{j}\right\}_{j \in \mathbb{N}_{0}}$ such that

$$
0=\lambda_{0}<\lambda_{1} \leq \lambda_{2} \leq \cdots \leq \lambda_{j} \leq \cdots, \quad \lambda_{j} \rightarrow \infty .
$$

Note that the first eigenfunction is a constant, i.e., $e_{0}=|D|^{-\frac{1}{2}}$ and $\left\{e_{j}\right\}_{j \in \mathbb{N}}$ forms an orthonormal basis of $\dot{H}$. By the spectral theory, we can define the fractional powers of $A$ on $\dot{H}$ in a simple way, e.g., $A^{\alpha} v=\sum_{j=1}^{\infty} \lambda_{j}^{\alpha}\left(v, e_{j}\right) e_{j}, \alpha \in \mathbb{R}$. Define the inner product $(\cdot, \cdot)_{\alpha}$ and the associated norm $|\cdot|_{\alpha}:=\left\|A^{\frac{\alpha}{2}} \cdot\right\|$, given by

$$
|v|_{\alpha}=\left\|A^{\frac{\alpha}{2}} v\right\|=\left(\sum_{j=1}^{\infty} \lambda_{j}^{\alpha}\left|\left(v, e_{j}\right)\right|^{2}\right)^{\frac{1}{2}}, \quad(v, w)_{\alpha}=\sum_{j=1}^{\infty} \lambda_{j}^{\alpha}\left(v, e_{j}\right)\left(w, e_{j}\right), \quad \alpha \in \mathbb{R} .
$$

Then we define the following function spaces

$$
\dot{H}^{\alpha}:=\operatorname{dom}\left(A^{\frac{\alpha}{2}}\right)=\left\{v \in \dot{H}:|v|_{\alpha}<\infty\right\}, \quad \alpha \geq 0 .
$$

Then $\dot{H}^{0}=\dot{H}$. For negative order $-\alpha<0$ we define $\dot{H}^{-\alpha}$ by taking the closure of $\dot{H}$ with respect to $|\cdot|_{-\alpha}$. It is known that for integer $\alpha \geq 0, \dot{H}^{\alpha}$ is a subspace of $H^{\alpha}(D) \cap \dot{H}$ characterized by certain boundary conditions. Additionally, the norm $|\cdot|_{\alpha}$ is equivalent on $\dot{H}^{\alpha}$ to the standard Sobolev norm $\|\cdot\|_{H^{\alpha}(D)}$ for $\alpha=1,2$.

Thanks to (2.2), the operator $-A^{2}$ can generate an analytic semigroup $E(t)=e^{-t A^{2}}$ on $H$ and

$$
E(t) v=e^{-t A^{2}} v=\sum_{j=1}^{\infty} e^{-t \lambda_{j}^{2}}\left(v, e_{j}\right) e_{j}+\left(v, e_{0}\right) e_{0}=P e^{-t A^{2}} v+(I-P) v, \quad v \in H .
$$

By expansion in terms of the eigenbasis of $A$ and using the Parseval identity, one can easily obtain

$$
\begin{aligned}
\left\|A^{\mu} E(t)\right\|_{\mathcal{L}(\dot{H})} & \leq C t^{-\frac{\mu}{2}}, t>0, \mu \geq 0, \\
\left\|A^{-\nu}(I-E(t))\right\|_{\mathcal{L}(\dot{H})} & \leq C t^{\frac{\nu}{2}}, \quad t \geq 0, \nu \in[0,2], \\
\int_{\tau_{1}}^{\tau_{2}}\left\|A^{\varrho} E(s) v\right\|^{2} \mathrm{~d} s & \leq C\left(\tau_{2}-\tau_{1}\right)^{1-\varrho}\|v\|^{2}, \forall v \in \dot{H}, \varrho \in[0,1], \tau_{2} \geq \tau_{1} \geq 0, \\
\left\|A^{2 \rho} \int_{\tau_{1}}^{\tau_{2}} E\left(\tau_{2}-\sigma\right) v \mathrm{~d} \sigma\right\| & \leq C\left(\tau_{2}-\tau_{1}\right)^{1-\rho}\|v\|, \forall v \in \dot{H}, \rho \in[0,1], \tau_{2} \geq \tau_{1} \geq 0 .
\end{aligned}
$$

Throughout the paper, $C$ denotes a generic positive constant that may change from line to line. The next assumption specifies the nonlinearity of the considered equation.

Assumption 2.2. (Nonlinearity) Let $F: L_{6}(D ; \mathbb{R}) \rightarrow H$ be a deterministic mapping given by

$$
F(v)(x)=f(v(x))=v^{3}(x)-v(x), \quad x \in D, v \in L_{6}(D ; \mathbb{R}) .
$$


It is easy to check that, for any $v, \psi, \psi_{1}, \psi_{2} \in L_{6}(D)$,

$$
\begin{aligned}
\left(F^{\prime}(v)(\psi)\right)(x) & =f^{\prime}(v(x)) \psi(x)=\left(3 v^{2}(x)-1\right) \psi(x), \quad x \in D, \\
\left(F^{\prime \prime}(v)\left(\psi_{1}, \psi_{2}\right)\right)(x) & =f^{\prime \prime}(v(x)) \psi_{1}(x) \psi_{2}(x)=6 v(x) \psi_{1}(x) \psi_{2}(x), \quad x \in D .
\end{aligned}
$$

Moreover, there exists a constant $C$ such that

$$
\begin{aligned}
-(F(u)-F(v), u-v) \leq\|u-v\|^{2}, & u, v \in L_{6}(D), \\
\|F(u)-F(v)\| \leq C\|u-v\|\left(1+\|u\|_{V}^{2}+\|v\|_{V}^{2}\right), & u, v \in V,
\end{aligned}
$$

where by $V:=C(D ; \mathbb{R})$ we denote a Banach space of continuous functions with a usual norm. In order to make the solution $X$ preserve the total mass, that is, $(I-P) X(t)=(I-P) X_{0}$, we assume the average of the Wiener process to be zero.

Assumption 2.3. (Noise process) Let $\{W(t)\}_{t \in[0, T]}$ be a standard $\dot{H}$-valued $Q$-Wiener process on the stochastic basis $\left(\Omega, \mathcal{F}, \mathbb{P},\left\{\mathcal{F}_{t}\right\}_{t \in[0, T]}\right)$, where the covariance operator $Q \in \mathcal{L}(\dot{H})$ is bounded, self-adjoint and positive semi-definite, satisfying

$$
\left\|A^{\frac{\gamma-2}{2}} Q^{\frac{1}{2}}\right\|_{\mathcal{L}_{2}}<\infty, \text { for some } \gamma \in[3,4] .
$$

Assumption 2.4. (Initial data) Let $X_{0}: \Omega \rightarrow \dot{H}$ be $\mathcal{F}_{0} / \mathcal{B}(\dot{H})$-measurable and satisfy, for a sufficiently large number $p_{0} \in \mathbb{N}$,

$$
\mathbf{E}\left[\left|X_{0}\right|_{\gamma}^{p_{0}}\right]<\infty
$$

where $\gamma \in[3,4]$ is the parameter coming from (2.11).

2.2. Regularity results of the model. This part is devoted to the well-posedness of the underlying problem (1.2) and the space-time regularity properties of the mild solution. Existence, uniqueness, and regularity of weak and mild solutions to (1.2) have been studied in [14, 27. The relevant result is stated as follows.

Theorem 2.1. If Assumptions 2.1 2.4 are valid, then the problem (1.2) admits a weak solution $X(t)$, which is almost surely continuous and satisfies the equation

$$
\begin{aligned}
\langle X(t), v\rangle-\left\langle X_{0}, v\right\rangle+\int_{0}^{t}\left\langle X(s), A^{2} v\right\rangle & +\langle F(X(s)), A v\rangle \mathrm{d} s \\
= & \langle W(t), v\rangle, \text { a.s. }, \quad \forall v \in \dot{H}^{4}=\operatorname{dom}\left(A^{2}\right), t \in[0, T] .
\end{aligned}
$$

In addition, the weak solution $X(t)$ is also a mild solution, given by (1.4), satisfying

$$
\sup _{t \in[0, T]}\|X(t)\|_{L^{p}\left(\Omega ; \dot{H}^{1}\right)}<\infty, \quad \forall p \geq 1 .
$$

To validate (2.13), one can simply adapt the proof of [27, Theorem 3.1], where it was shown that $\mathbf{E}[J(X(t))]+\mathbf{E}\left[\int_{0}^{t} J^{\prime}(X(s)) \mathrm{d} s\right] \leq C(t)$ by introducing the following Lyapunov functional

$$
J(u)=\frac{1}{2}\|\nabla u\|^{2}+\int_{D} \Phi(u) \mathrm{d} x, u \in \dot{H}^{1} .
$$

Here $\Phi(s):=\frac{1}{4}\left(s^{2}-1\right)^{2}$ is a primitive of $f(s)=s^{3}-s$. Evidently, the above estimate (2.13) together with the embedding inequality $\dot{H}^{1} \subset L_{6}(D)$ suffices to ensure

$$
\sup _{s \in[0, T]}\|F(X(s))\|_{L^{p}(\Omega ; H)} \leq C\left(1+\left(\sup _{s \in[0, T]}\|X(s)\|_{L^{3 p}\left(\Omega ; \dot{H}^{1}\right)}\right)^{3}\right)<\infty
$$


and similarly

$$
\sup _{s \in[0, T]}\left\|f^{\prime}(X(s))\right\|_{L^{p}\left(\Omega ; L_{3}\right)}+\sup _{s \in[0, T]}\left\|f^{\prime \prime}(X(s))\right\|_{L^{p}\left(\Omega ; L_{6}\right)}<\infty .
$$

Furthermore, one can show further properties of the mild solution as follows.

Theorem 2.2. Let Assumptions 2.1]2.4 be fulfilled. Then the unique mild solution (1.4) enjoys the following regularity properties,

$$
\begin{aligned}
& \sup _{t \in[0, T]}\|X(t)\|_{L^{p}\left(\Omega ; \dot{H}^{\gamma}\right)}<\infty, \forall p \geq 1, \\
&\|X(t)-X(s)\|_{L^{p}\left(\Omega ; \dot{H}^{\beta}\right)} \leq C|t-s|^{\min \left\{\frac{1}{2}, \frac{\gamma-\beta}{4}\right\}}, \quad \forall p \geq 1,0 \leq s<t \leq T, \beta \in[0, \gamma],
\end{aligned}
$$

where $\gamma \in[3,4]$ comes from Assumption 2.3.

Here and below $C$ is a generic positive constant that is also dependent of $\gamma, p, T$ and the initial data, but independent of the discretization parameters $h$ and $k$. To prove the theorem, we introduce some basic inequalities. Recall first the following embedding inequalities,

$$
\dot{H}^{1} \subset L_{6}(D) \quad \text { and } \quad \dot{H}^{\delta} \subset C(D ; \mathbb{R}), \quad \text { for } \delta>\frac{d}{2}, d \in\{1,2,3\} .
$$

With (2.1) and (2.19) at hand, one can further derive, for any $\delta>\frac{d}{2}$ and any $x \in L_{2}(D)$,

$$
\left\|A^{-\frac{\delta}{2}} P x\right\|=\sup _{v \in \dot{H}} \frac{\left|\left(P x, A^{-\frac{\delta}{2}} v\right)\right|}{\|v\|} \leq \sup _{v \in \dot{H}} \frac{\|P x\|_{L_{1}}\left\|A^{-\frac{\delta}{2}} v\right\|_{V}}{\|v\|} \leq C \sup _{v \in \dot{H}} \frac{\|P x\|_{L_{1}}\|v\|}{\|v\|} \leq C\|x\|_{L_{1}} .
$$

Similarly, one can see that, for any $x \in L_{\frac{6}{5}}(D)$,

$$
\left\|A^{-\frac{1}{2}} P x\right\|=\sup _{v \in \dot{H}} \frac{\left|\left(P x, A^{-\frac{1}{2}} v\right)\right|}{\|v\|} \leq \sup _{v \in \dot{H}} \frac{\|P x\|_{L_{\frac{6}{5}}}\left\|A^{-\frac{1}{2}} v\right\|_{L_{6}}}{\|v\|} \leq C \sup _{v \in \dot{H}} \frac{\|x\|_{L_{\frac{6}{g}}}\|v\|}{\|v\|} \leq C\|x\|_{L_{\frac{6}{5}}} .
$$

Since the norm $|\cdot|_{2}$ is equivalent on $\dot{H}^{2}$ to the standard Sobolev norm $\|\cdot\|_{H^{2}(D)}$ and $H^{2}(D)$ is an algebra, one can find a constant $C>0$ such that, for any $f, g \in \dot{H}^{2}$,

$$
\|f g\|_{H^{2}(D)} \leq C\|f\|_{H^{2}(D)}\|g\|_{H^{2}(D)} \leq C|f|_{2}|g|_{2} .
$$

Proof of Theorem 2.2. We start by proving a preliminary spatial-temporal regularity of the mild solution. Using (2.4) with $\mu=0, \frac{\delta_{0}+2}{2}$, (2.6) with $\varrho=1$ and (2.15), one can observe that, for any fixed $\frac{3}{2}<\delta_{0}<2$,

$$
\begin{aligned}
\|X(t)\|_{L^{p}\left(\Omega ; \dot{H}^{\delta_{0}}\right)} \leq & \left\|E(t) X_{0}\right\|_{L^{p}\left(\Omega ; \dot{H}^{\delta_{0}}\right)} \\
& +\int_{0}^{t}\|E(t-s) A P F(X(s))\|_{L^{p}\left(\Omega ; \dot{H}^{\delta_{0}}\right)} \mathrm{d} s+\left(\int_{0}^{t}\left\|A^{\frac{\delta_{0}}{2}} E(t-r) Q^{\frac{1}{2}}\right\|_{\mathcal{L}_{2}}^{2} \mathrm{~d} r\right)^{\frac{1}{2}} \\
\leq & C\left(\left\|X_{0}\right\|_{L^{p}\left(\Omega ; \dot{H}^{\delta_{0}}\right)}+\int_{0}^{t}(t-s)^{-\frac{\delta_{0}+2}{4}}\|F(X(s))\|_{L^{p}(\Omega ; H)} \mathrm{d} s+\left\|A^{\frac{\delta_{0}-2}{2}} Q^{\frac{1}{2}}\right\|_{\mathcal{L}_{2}}\right) \\
\leq & C\left\|X_{0}\right\|_{L^{p}\left(\Omega ; \dot{H}^{\delta_{0}}\right)}+C \sup _{s \in[0, T]}\|F(X(s))\|_{L^{p}(\Omega ; H)}+C\left\|Q^{\frac{1}{2}}\right\|_{\mathcal{L}_{2}}<\infty,
\end{aligned}
$$

where we also used the Burkholder-Davis-Gundy-type inequality and the fact $A v=A P v$, for any $v \in H$. Concerning the temporal regularity of the mild solution, we utilize (2.4)-(2.6), (2.15) and the 
Burkholder-Davis-Gundy-type inequality to get, for $\beta \in\left[0, \delta_{0}\right]$ with $\frac{3}{2}<\delta_{0}<2$,

$$
\begin{aligned}
\| X(t)- & X(s)\left\|_{L^{p}\left(\Omega ; \dot{H}^{\beta}\right)} \leq\right\|(E(t-s)-I) X(s) \|_{L^{p}\left(\Omega ; \dot{H}^{\beta}\right)} \\
& +\int_{s}^{t}\|E(t-r) A P F(X(r))\|_{L^{p}\left(\Omega ; \dot{H}^{\beta}\right)} \mathrm{d} r+C\left(\int_{s}^{t}\left\|A^{\frac{\beta}{2}} E(t-r) Q^{\frac{1}{2}}\right\|_{\mathcal{L}_{2}}^{2} \mathrm{~d} r\right)^{\frac{1}{2}} \\
\leq & C(t-s)^{\frac{\delta_{0}-\beta}{4}}\left(\|X(s)\|_{L^{p}\left(\Omega ; \dot{H}^{\left.\delta_{0}\right)}\right.}+\left\|A^{\frac{\delta_{0}-2}{2}} Q^{\frac{1}{2}}\right\|_{\mathcal{L}_{2}}\right)+C \int_{s}^{t}(t-r)^{-\frac{2+\beta}{4}}\|F(X(r))\|_{L^{p}(\Omega ; H)} \mathrm{d} r \\
\leq & C(t-s)^{\frac{\delta_{0}-\beta}{4}}\left(\sup _{s \in[0, T]}\|X(s)\|_{L^{p}\left(\Omega ; \dot{H}^{\delta_{0}}\right)}+\left\|Q^{\frac{1}{2}}\right\|_{\mathcal{L}_{2}}+(t-s)^{\frac{2-\delta_{0}}{4}} \sup _{s \in[0, T]}\|F(X(s))\|_{L^{p}(\Omega ; H)}\right) \\
\leq & C(t-s)^{\frac{\delta_{0}-\beta}{4}}
\end{aligned}
$$

where we also used the fact that $(t-s)^{\frac{2-\delta_{0}}{4}} \leq T^{\frac{2-\delta_{0}}{4}}$ for $\frac{3}{2}<\delta_{0}<2,0 \leq s<t \leq T$ and thus the final constant $C$ depends on $T$. Next we show an improved spatial regularity of the mild solution. First, the above two estimates and (2.21) imply,

$$
\begin{aligned}
& \left.\left\|A^{-\frac{1}{2}} P(F(X(s))-F(X(t)))\right\|_{L^{p}(\Omega ; \dot{H})} \leq C\|F(X(s))-F(X(t))\|_{L^{p}\left(\Omega ; L_{\frac{6}{5}}\right.}\right) \\
& \quad \leq C\|X(s)-X(t)\|_{L^{2 p}(\Omega ; \dot{H})}\left(1+\sup _{s \in[0, T]}\|X(s)\|_{L^{4 p}\left(\Omega ; L_{6}\right)}^{2}\right) \\
& \quad \leq C|t-s|^{\frac{\delta_{0}}{4}}, \quad \forall \delta_{0} \in\left(\frac{3}{2}, 2\right) .
\end{aligned}
$$

Combining this with (2.15), (2.4)-(2.7) and the Burkholder-Davis-Gundy-type inequality shows, for $\delta_{0} \in\left(\frac{3}{2}, 2\right)$ and $t \in[0, T]$,

$$
\begin{aligned}
\|X(t)\|_{L^{p}\left(\Omega ; \dot{H}^{2}\right)} \leq & \left\|E(t) X_{0}\right\|_{L^{p}\left(\Omega ; \dot{H}^{2}\right)}+\int_{0}^{t}\left\|E(t-s) A^{2} P(F(X(s))-F(X(t)))\right\|_{L^{p}(\Omega ; \dot{H})} \mathrm{d} s \\
& +\left\|\int_{0}^{t} E(t-s) A^{2} P F(X(t)) \mathrm{d} s\right\|_{L^{p}(\Omega ; \dot{H})}+\left\|\int_{0}^{t} A E(t-s) \mathrm{d} W(s)\right\|_{L^{p}(\Omega ; \dot{H})} \\
\leq & C\left\|X_{0}\right\|_{L^{p}\left(\Omega ; \dot{H}^{2}\right)}+C \int_{0}^{t}(t-s)^{-\frac{5}{4}}\left\|A^{-\frac{1}{2}} P(F(X(s))-F(X(t)))\right\|_{L^{p}(\Omega ; \dot{H})} \mathrm{d} s \\
& +C\|F(X(t))\|_{L^{p}(\Omega ; H)}+C\left(\int_{0}^{t}\left\|A E(t-s) Q^{\frac{1}{2}}\right\|_{\mathcal{L}_{2}}^{2} \mathrm{~d} s\right)^{\frac{1}{2}} \\
\leq & C\left(\left\|X_{0}\right\|_{L^{p}\left(\Omega ; \dot{H}^{2}\right)}+\int_{0}^{t}(t-s)^{\frac{\delta_{0}-5}{4}} \mathrm{~d} s+\sup _{s \in[0, T]}\|F(X(s))\|_{L^{p}(\Omega ; H)}+\left\|Q^{\frac{1}{2}}\right\|_{\mathcal{L}_{2}}\right) \\
< &
\end{aligned}
$$

Taking the above estimate and (2.22) into account, one deduces

$$
\sup _{s \in[0, T]}\|P F(X(s))\|_{L^{p}\left(\Omega ; \dot{H}^{2}\right)} \leq C \sup _{s \in[0, T]}\|P F(X(s))\|_{L^{p}\left(\Omega ; H^{2}(D)\right)} \leq C\left(1+\sup _{s \in[0, T]}\|X(s)\|_{L^{3 p}\left(\Omega ; \dot{H}^{2}\right)}^{3}\right)<\infty,
$$

where we recalled $P v=v-|D|^{-1} \int_{D} v \mathrm{~d} x, v \in H$. Then, repeating the same lines in the proof of (2.23) and (2.24) can readily result in

$$
\sup _{s \in[0, T]}\|X(s)\|_{L^{p}\left(\Omega ; \dot{H}^{3}\right)}<\infty
$$


and

$$
\|X(t)-X(s)\|_{L^{p}\left(\Omega ; \dot{H}^{2}\right)} \leq C|t-s|^{\frac{1}{4}}, \quad \forall 0 \leq s<t \leq T .
$$

Further, the above estimates together with (2.22) and (2.19) imply

$$
\begin{aligned}
\|P(F(X(t))-F(X(s)))\|_{L^{p}\left(\Omega ; \dot{H}^{2}\right)} & \leq C\|P(F(X(t))-F(X(s)))\|_{L^{p}\left(\Omega ; H^{2}(D)\right)} \\
& \leq C\|X(t)-X(s)\|_{L^{2 p}\left(\Omega ; \dot{H}^{2}\right)}\left(1+\sup _{s \in[0, T]}\|X(s)\|_{L^{4 p}\left(\Omega ; \dot{H}^{2}\right)}^{2}\right) \\
& \leq C|t-s|^{\frac{1}{4}} .
\end{aligned}
$$

Bearing this in mind and applying (2.4) with $\mu=\frac{\beta}{2}$, (2.7) with $\rho=\frac{\beta}{4}$ and (2.27), one can prove, for $\beta \in[0,4]$ and $0 \leq s<t \leq T$,

$$
\begin{aligned}
\left\|\int_{s}^{t} E(t-r) A P F(X(r)) \mathrm{d} r\right\|_{L^{p}\left(\Omega ; \dot{H}^{\beta}\right)} & \int_{s}^{t}\left\|E(t-r) A^{\frac{\beta}{2}}\right\|_{\mathcal{L}(\dot{H})}\|P(F(X(r))-F(X(t)))\|_{L^{p}\left(\Omega ; \dot{H}^{2}\right)} \mathrm{d} r \\
& +\left\|\int_{s}^{t} E(t-r) A^{\frac{\beta+2}{2}} P F(X(t)) \mathrm{d} r\right\|_{L^{p}(\Omega ; \dot{H})} \\
\leq & C \int_{s}^{t}(t-r)^{\frac{1-\beta}{4}} \mathrm{~d} r+C(t-s)^{\frac{4-\beta}{4}}\|P F(X(t))\|_{L^{p}\left(\Omega ; \dot{H}^{2}\right)} \\
\leq & C(t-s)^{\frac{4-\beta}{4}} .
\end{aligned}
$$

This together with (2.4)-(2.7) and the Burkholder-Davis-Gundy inequality gives

$$
\begin{aligned}
\|X(t)\|_{L^{p}\left(\Omega ; \dot{H}^{\gamma}\right)} \leq & \left\|E(t) X_{0}\right\|_{L^{p}\left(\Omega ; \dot{H}^{\gamma}\right)}+\left\|\int_{0}^{t} E(t-s) A P F(X(s)) \mathrm{d} s\right\|_{L^{p}\left(\Omega ; \dot{H}^{\gamma}\right)} \\
& +\left(\int_{0}^{t}\left\|A^{\frac{\gamma}{2}} E(t-s) Q^{\frac{1}{2}}\right\|_{\mathcal{L}_{2}}^{2} \mathrm{~d} s\right)^{\frac{1}{2}} \\
\leq & C\left(\left\|X_{0}\right\|_{L^{p}\left(\Omega ; \dot{H}^{\gamma}\right)}+t^{\frac{4-\gamma}{4}}+\left\|A^{\frac{\gamma-2}{2}} Q^{\frac{1}{2}}\right\|_{\mathcal{L}_{2}}\right)<\infty, \quad \forall t \in[0, T] .
\end{aligned}
$$

This confirms (2.17). To prove (2.18), we use (2.29), (2.30), (2.5) with $\nu=\frac{\gamma-\beta}{2}$ and (2.6) with $\varrho=\max \left\{\frac{\beta+2-\gamma}{2}, 0\right\}$ to derive that

$$
\begin{aligned}
& \|X(t)-X(s)\|_{L^{p}\left(\Omega ; \dot{H}^{\beta}\right)} \leq\|(E(t-s)-I) X(s)\|_{L^{p}\left(\Omega ; \dot{H}^{\beta}\right)} \\
& +\left\|\int_{s}^{t} E(t-r) A P F(X(r)) \mathrm{d} r\right\|_{L^{p}\left(\Omega ; \dot{H}^{\beta}\right)}+C\left(\int_{s}^{t}\left\|A^{\frac{\beta-\gamma+2}{2}} E(t-r) A^{\frac{\gamma-2}{2}} Q^{\frac{1}{2}}\right\|_{\mathcal{L}_{2}}^{2} \mathrm{~d} r\right)^{\frac{1}{2}} \\
& \leq C(t-s)^{\frac{\gamma-\beta}{4}}\|X(s)\|_{L^{p}\left(\Omega ; \dot{H}^{\gamma}\right)}+C(t-s)^{\frac{4-\beta}{4}}+C(t-s)^{\frac{1}{2}\left[1-\max \left\{\frac{\beta+2-\gamma}{2}, 0\right\}\right]}\left\|A^{\frac{\gamma-2}{2}} Q^{\frac{1}{2}}\right\|_{\mathcal{L}_{2}} \\
& \leq C(t-s)^{\frac{\gamma-\beta}{4}}\|X(s)\|_{L^{p}\left(\Omega ; \dot{H}^{\gamma}\right)}+C T^{\frac{4-\gamma}{4}}(t-s)^{\frac{\gamma-\beta}{4}}+C(t-s)^{\min \left\{\frac{\gamma-\beta}{4}, \frac{1}{2}\right\}}\left\|A^{\frac{\gamma-2}{2}} Q^{\frac{1}{2}}\right\|_{\mathcal{L}_{2}} \\
& \leq C(t-s)^{\min \left\{\frac{\gamma-\beta}{4}, \frac{1}{2}\right\}},
\end{aligned}
$$

as required. This thus finishes the proof of the theorem.

As a direct consequence of Theorem 2.2 the following lemma holds.

Lemma 2.3. Let Assumptions 2.1 2.4 be fulfilled. Then the following results hold

$$
\sup _{s \in[0, T]}\left\|A^{\frac{1}{2}} P F^{\prime}(X(s)) A^{-\frac{1}{2}} P v\right\|_{L^{p}(\Omega ; \dot{H})} \leq C\left(1+\sup _{s \in[0, T]}\|X(s)\|_{L^{2 p}\left(\Omega ; \dot{H}^{2}\right)}^{2}\right)\|v\|_{L_{6}}, \forall p \geq 1, v \in L_{6}(D),
$$




$$
\|P(F(X(t))-F(X(s)))\|_{L^{p}\left(\Omega ; \dot{H}^{\beta}\right)} \leq C|t-s|^{\min \left\{\frac{1}{2}, \frac{\gamma-\beta}{4}\right\}}, \forall p \geq 1,0 \leq s<t \leq T, \beta \in\{0,1,2\},
$$

where $\gamma \in[3,4]$ comes from Assumption 2.3.

Proof of Lemma 2.3. Note first that $f^{\prime}(v)=3 v^{2}-1, v \in \mathbb{R}$. Thus, from (2.19), (2.17) and Hölder's inequality, it follows that, for any $v \in L_{6}(D)$,

$$
\begin{aligned}
& \left\|A^{\frac{1}{2}} P F^{\prime}(X(s)) A^{-\frac{1}{2}} P v\right\|_{L^{p}(\Omega ; \dot{H})} \leq\left\|\nabla\left(3 X^{2}(s)-1\right) A^{-\frac{1}{2}} P v\right\|_{L^{p}(\Omega ; H)}+\left\|\left(3 X^{2}(s)-1\right) \nabla A^{-\frac{1}{2}} P v\right\|_{L^{p}(\Omega ; H)} \\
& \quad \leq C\left(1+\|X(s) \nabla X(s)\|_{L^{p}\left(\Omega ; L_{3}\right)}+\left\|X^{2}(s)\right\|_{L^{p}(\Omega ; V)}\right)\left(\left\|A^{-\frac{1}{2}} P v\right\|_{L_{6}}+\|v\|_{L_{6}}\right) \\
& \quad \leq C\left(1+\|X(s)\|_{L^{2 p}\left(\Omega ; \dot{H}^{2}\right)}^{2}\right)\|v\|_{L_{6}} .
\end{aligned}
$$

To validate (2.33), we first recall (2.18) and (2.28) to attain the desired assertion for the case $\beta=2$. With regard to the case $\beta=1$, one can apply (2.20), Sobolev's inequality $\|u\|_{L_{3}} \leq C\|u\|_{H^{1}(D)} \leq C|u|_{1}$ and Hölder's inequality to show

$$
\begin{aligned}
\| A^{\frac{1}{2}} & P(F(X(t))-F(X(s)))\|\leq\| \nabla(F(X(t, \cdot))-F(X(s, \cdot))) \| \\
\leq & \left\|(X(t, \cdot)-X(s, \cdot)) \cdot \nabla\left(X^{2}(t, \cdot)+X(t, \cdot) X(s, \cdot)+X^{2}(s, \cdot)\right)\right\| \\
& +\left\|\nabla(X(t, \cdot)-X(s, \cdot)) \cdot\left(X^{2}(t, \cdot)+X(t, \cdot) X(s, \cdot)+X^{2}(s, \cdot)\right)\right\|+|X(t)-X(s)|_{1} \\
\leq & C\|X(t)-X(s)\|_{L_{6}}\left(\|\nabla X(t)\|_{L_{3}}+\|\nabla X(s)\|_{L_{3}}\right)\left(\|X(t)\|_{V}+\|X(s)\|_{V}\right) \\
& +C|X(t)-X(s)|_{1}\left(1+\|X(t)\|_{V}^{2}+\|X(s)\|_{V}^{2}\right) \\
\leq & C|X(t)-X(s)|_{1}\left(1+|X(t)|_{2}^{2}+|X(s)|_{2}^{2}\right) .
\end{aligned}
$$

Further, combining this with (2.18) enables us to obtain

$$
\begin{aligned}
\|P(F(X(t))-F(X(s)))\|_{L^{p}\left(\Omega ; \dot{H}^{1}\right)} & \leq C\|X(t)-X(s)\|_{L^{2 p}\left(\Omega ; \dot{H}^{1}\right)}\left(1+\|X(t)\|_{L^{4 p}\left(\Omega ; \dot{H}^{2}\right)}^{2}+\|X(s)\|_{L^{4 p}\left(\Omega ; \dot{H}^{2}\right)}^{2}\right) \\
& \leq C|t-s|^{\min \left\{\frac{1}{2}, \frac{\gamma-1}{4}\right\}},
\end{aligned}
$$

verifying (2.33) for the case $\beta=1$. Similarly, one can easily deduce (2.33) for $\beta=0$, by taking (2.10) and (2.18) into account. Hence the proof of this lemma is complete.

3. The finite element spatial semi-discretization. In this section, we examine the finite element spatial semi-discretization of the CHC equation and show uniform-in-time moment bounds of the solution to the semi-discrete problem, which will be used later for the convergence analysis.

3.1. Basic elements of the finite element spatial discretization. Before coming to the semi-discrete finite element method (FEM) for (1.2), we make the following assumptions.

Assumption 3.1. Suppose that the spatial domain $D \subset \mathbb{R}^{d}, d \in\{1,2,3\}$ has polygonal boundary $\partial D$. The triangulations $\left\{\mathcal{T}_{h}\right\}_{h>0}$ of $D$ with maximal mesh size $h$, are assumed to be quasi-uniform. Let $\left\{V_{h}\right\}_{h>0} \subset H^{1}(D)$ be the space of continuous functions that are piecewise polynomials of degree at most $r-1$ over $\mathcal{T}_{h}$, for $r \in\{2,3,4\}$ in dimension $d=1$ and $r=2$ in dimension $d \in\{2,3\}$.

Furthermore, we define $\dot{V}_{h}=P V_{h}$ by

$$
\dot{V}_{h}=\left\{v_{h} \in V_{h}: \int_{D} v_{h} \mathrm{~d} x=0\right\},
$$

and introduce a discrete Laplace operator $A_{h}: V_{h} \rightarrow V_{h}$ defined by

$$
\left(A_{h} v_{h}, \chi_{h}\right)=a\left(v_{h}, \chi_{h}\right):=\left(\nabla v_{h}, \nabla \chi_{h}\right), \quad \forall v_{h} \in V_{h}, \chi_{h} \in V_{h} .
$$


The operator $A_{h}$ is selfadjoint, positive semidefinite on $V_{h}$ and positive definite on $\dot{V}_{h}$, and has an orthonormal eigenbasis $\left\{e_{j, h}\right\}_{j=0}^{\mathcal{N}_{h}}$ in $V_{h}$ with corresponding eigenvalues $\left\{\lambda_{j, h}\right\}_{j=0}^{\mathcal{N}_{h}}$, satisfying

$$
0=\lambda_{0, h}<\lambda_{1, h} \leq \cdots \leq \lambda_{j, h} \leq \cdots \leq \lambda_{\mathcal{N}_{h}, h},
$$

where $\mathcal{N}_{h}:=\operatorname{dim}\left(V_{h}\right)$ and $e_{0, h}=e_{0}=|D|^{-\frac{1}{2}}$. Also, we introduce a discrete norm on $\dot{V}_{h}$, defined by

$$
\left|v_{h}\right|_{\alpha, h}=\left\|A_{h}^{\frac{\alpha}{2}} v_{h}\right\|=\left(\sum_{j=1}^{\mathcal{N}_{h}} \lambda_{j, h}^{\alpha}\left|\left(v_{h}, e_{j, h}\right)\right|^{2}\right)^{\frac{1}{2}}, v_{h} \in \dot{V}_{h}, \alpha \in \mathbb{R},
$$

which corresponds to the discrete inner product $(v, w)_{\alpha, h}:=\left(A_{h}^{\frac{\alpha}{2}} v, A_{h}^{\frac{\alpha}{2}} w\right), \forall v, w \in \dot{V}_{h}$. Note that

$$
\left|v_{h}\right|_{1}=\left\|A^{\frac{1}{2}} v_{h}\right\|=\left\|\nabla v_{h}\right\|=\left\|A_{h}^{\frac{1}{2}} v_{h}\right\|=\left|v_{h}\right|_{1, h}, \quad v_{h} \in \dot{V}_{h} .
$$

In addition, we introduce a Riesz representation operator $R_{h}: \dot{H}^{1} \rightarrow \dot{V}_{h}$ defined by

$$
a\left(R_{h} v, \chi_{h}\right)=a\left(v, \chi_{h}\right), \quad \forall v \in \dot{H}^{1}, \chi_{h} \in \dot{V}_{h},
$$

and a generalized projection operator $P_{h}: H \rightarrow V_{h}$ given by

$$
\left(P_{h} v, \chi_{h}\right)=\left(v, \chi_{h}\right), \quad \forall v \in H, \chi_{h} \in V_{h} .
$$

It is easy to see that $P_{h}$ is also a projection operator from $\dot{H}$ to $\dot{V}_{h}$ and

$$
P_{h} A=A_{h} R_{h} .
$$

Owing to Assumption 3.1, we have the following error bounds for the operators $R_{h}$ and $P_{h}$ (cf. [38 Chapter 1] and [30, (2.3)]),

$$
\left|\left(I-R_{h}\right) v\right|_{i}+\left|\left(I-P_{h}\right) v\right|_{i} \leq C h^{\beta-i}|v|_{\beta}, \forall v \in \dot{H}^{\beta}, i=0,1, \beta \in[1, r],
$$

where $r \in\{2,3,4\}$ for $d=1$ and $r=2$ for $d \in\{2,3\}$. We mention that only $r=2$ is considered for $d \in\{2,3\}$, because for higher-order elements, i.e., $r \in\{3,4\}$, the situation becomes very complicated in high dimension $d \in\{2,3\}$. Also, the quasi-uniform mesh $\mathcal{T}_{h}$ ensures that $P_{h}$ is bounded with respect to the $\dot{H}^{1}$ and $L_{4}$ norms and that we have an inverse bound for $A_{h}$,

$$
\begin{aligned}
\left\|P_{h} v\right\|_{L_{4}} & \leq C\|v\|_{L_{4}}, \quad \forall v \in L_{4}, \\
\left|P_{h} v\right|_{1} & \leq C|v|_{1}, \quad \forall v \in \dot{H}^{1}, \\
\left\|A_{h} v_{h}\right\| & \leq C h^{-2}\left\|v_{h}\right\|, \quad \forall v_{h} \in V_{h} .
\end{aligned}
$$

These three inequalities have been also claimed in [27, (2.12)]. The inverse inequality (3.9) together with (3.6) helps us to infer

$$
\left\|A_{h} P_{h} v\right\| \leq\left\|A_{h} P_{h}\left(I-R_{h}\right) v\right\|+\left\|P_{h} A v\right\| \leq C h^{-2}\left\|\left(I-R_{h}\right) v\right\|+C|v|_{2} \leq C|v|_{2}, \quad \forall v \in \dot{H}^{2} .
$$

Moreover, the operators $A$ and $A_{h}$ obey

$$
C_{1}\left\|A_{h}^{\frac{\alpha}{2}} P_{h} v\right\| \leq\left\|A^{\frac{\alpha}{2}} v\right\| \leq C_{2}\left\|A_{h}^{\frac{\alpha}{2}} P_{h} v\right\|, \quad \forall v \in \dot{H}^{\alpha}, \alpha \in[-1,1],
$$

and similarly to that in [38, Theorem $6.11,(6.91)]$,

$$
\left\|v_{h}\right\|_{V} \leq C\left\|A_{h} v_{h}\right\|, \quad \forall v_{h} \in \dot{V}_{h} .
$$

Combining (3.10) with (3.11) gives

$$
\left\|A_{h}^{\frac{\alpha}{2}} P_{h} v\right\| \leq C\left\|A^{\frac{\alpha}{2}} v\right\|, \quad \forall v \in \dot{H}^{\alpha}, \alpha \in[-1,2] .
$$


3.2. Moment bounds of the approximation. In this subsection, we come to the semi-discrete finite element approximation of the stochastic problem and provide some useful moment bounds for the semi-discrete approximations.

The semi-discrete finite element method for the problem (1.2) can be written as,

$$
\mathrm{d} X_{h}(t)+A_{h}\left(A_{h} X_{h}(t)+P_{h} F\left(X_{h}(t)\right)\right) \mathrm{d} t=P_{h} \mathrm{~d} W(t), \quad X_{h}(0)=P_{h} X_{0} .
$$

Similarly to (2.3), the analytic semigroup $E_{h}(t)$ generated by the discrete operator $-A_{h}^{2}$ can be given as follows,

$$
E_{h}(t) P_{h} v=e^{-t A_{h}^{2}} P_{h} v=\sum_{j=0}^{\mathcal{N}_{h}} e^{-t \lambda_{j, h}^{2}}\left(P_{h} v, e_{j, h}\right) e_{j, h}=P E_{h}(t) P_{h} v+(I-P) v .
$$

Since $\dot{V}_{h}$ is finite-dimensional and $F$ is a polynomial of particular structure and recalling $X_{0}, W(t)$ are $\dot{H}$-valued and thus $P_{h} X_{0}, P_{h} W(t)$ are $\dot{V}_{h}$-valued, one can easily check that the problem (3.14) admits a unique $\dot{V}_{h}$-valued solution $X_{h}(t)$, adapted, almost surely continuous, satisfying

$$
X_{h}(t)-P_{h} X_{0}+\int_{0}^{t}\left(A_{h}^{2} X_{h}(s)+A_{h} P_{h} F\left(X_{h}(s)\right)\right) \mathrm{d} s=P_{h} W(t),
$$

or equivalently in a mild solution form,

$$
X_{h}(t)=E_{h}(t) P_{h} X_{0}-\int_{0}^{t} E_{h}(t-s) A_{h} P_{h} F\left(X_{h}(s)\right) \mathrm{d} s+\int_{0}^{t} E_{h}(t-s) P_{h} \mathrm{~d} W(s) .
$$

Before presenting moment bounds of the approximations, we introduce a spatially discrete version of (2.4)-(2.7), which plays an important role in deriving the moment bounds of $X_{h}$.

Lemma 3.1. Under Assumptions 2.1, 3.1, the following estimates for $E_{h}$ hold,

$$
\begin{aligned}
\left\|A_{h}^{\mu} E_{h}(t) P_{h} v\right\| & \leq C t^{-\frac{\mu}{2}}\|v\|, \quad \forall \mu \geq 0, v \in \dot{H}, \\
\left\|A_{h}^{-\nu}\left(I-E_{h}(t)\right) P_{h} v\right\| & \leq C t^{\frac{\nu}{2}}\|v\|, \quad \forall \nu \in[0,2], v \in \dot{H}, \\
\left\|\int_{0}^{t} A_{h}^{2} E_{h}(s) P_{h} v \mathrm{~d} s\right\| & \leq C\|v\|, \quad \forall v \in \dot{H}, \\
\left(\int_{0}^{t}\left\|A_{h} E_{h}(s) P_{h} v\right\|^{2} \mathrm{~d} s\right)^{\frac{1}{2}} & \leq C\|v\|, \quad \forall v \in \dot{H} .
\end{aligned}
$$

Note that estimates (3.16), (3.19) can be found in 30, (2.1)-(2.2)]. Actually, thanks to the expansion of the eigenbasis of $A_{h}$ in $\dot{V}_{h}$ and the Parseval identity, one can follow standard arguments to derive these estimates. Next, we are ready to show the following moment bounds for the FEM approximation.

Theorem 3.2. Let $X_{h}(t)$ be the solution to (3.14). If Assumptions 2.1 2.4, 3.1 are valid, then

$$
\sup _{s \in[0, T]}\left\|A_{h} X_{h}(s)\right\|_{L^{p}(\Omega ; \dot{H})}+\left\|\int_{0}^{T}\left|A_{h} X_{h}(s)+P_{h} F\left(X_{h}(s)\right)\right|_{1}^{2} \mathrm{~d} s\right\|_{L^{p}(\Omega ; \mathbb{R})}<\infty, \quad \forall p \geq 1 .
$$

Proof of Theorem 3.2. A slight modification of the proof of [27, Theorem 3.1] enables us to obtain

$$
\begin{aligned}
& \left\|\sup _{s \in[0, T]} J\left(X_{h}(s)\right)\right\|_{L^{p}(\Omega ; \mathbb{R})}^{p}+\left\|\int_{0}^{T}\left|A_{h} X_{h}(s)+P_{h} F\left(X_{h}(s)\right)\right|_{1}^{2} \mathrm{~d} s\right\|_{L^{p}(\Omega ; \mathbb{R})}^{p} \\
& \quad \leq C\left(1+\left\|J\left(P_{h} X_{0}\right)\right\|_{L^{p}(\Omega ; \mathbb{R})}^{p}+\left\|\sup _{t \in[0, T]}\left|\int_{0}^{t}\left(A_{h} X_{h}(s)+P_{h} F\left(X_{h}(s)\right), P_{h} \mathrm{~d} W(s)\right)\right|^{p}\right\|_{L^{1}(\Omega ; \mathbb{R})}\right),
\end{aligned}
$$


where $J$ is defined by (2.14). Owing to (3.7) and (3.8), one knows $\left\|J\left(P_{h} X_{0}\right)\right\|_{L^{p}(\Omega ; \mathbb{R})}<\infty$. Further, with the aid of the Burkhoder-Davis-Gundy-type inequality, one can find that

$$
\begin{aligned}
\| & \sup _{s \in[0, T]} J\left(X_{h}(s)\right)\left\|_{L^{p}(\Omega ; \mathbb{R})}^{p}+\right\| \int_{0}^{T}\left|A_{h} X_{h}(s)+P_{h} F\left(X_{h}(s)\right)\right|_{1}^{2} \mathrm{~d} s \|_{L^{p}(\Omega ; \mathbb{R})}^{p} \\
& \leq C\left(1+\left\|\int_{0}^{T}\right\| Q^{\frac{1}{2}}\left(A_{h} X_{h}(s)+P_{h} F\left(X_{h}(s)\right)\right)\left\|^{2} \mathrm{~d} s\right\|_{L^{\frac{p}{2}}(\Omega ; \mathbb{R})}^{\frac{p}{2}}\right) \\
& \leq C\left(1+\left\|Q^{\frac{1}{2}}\right\|_{\mathcal{L}(\dot{H})}^{p}\left\|\int_{0}^{T}\right\| A_{h} X_{h}(s)+P_{h} F\left(X_{h}(s)\right)\left\|^{2} \mathrm{~d} s\right\|_{L^{\frac{p}{2}(\Omega ; \mathbb{R})}}^{\frac{p}{2}}\right) \\
& \leq C\left(1+\frac{\left\|Q^{\frac{1}{2}}\right\|_{\mathcal{L}(\dot{H})}^{2 p}}{2 \varepsilon}+\frac{\varepsilon}{2}\left\|\int_{0}^{T}\left|A_{h} X_{h}(s)+P_{h} F\left(X_{h}(s)\right)\right|_{1}^{2} \mathrm{~d} s\right\|_{L^{p}(\Omega ; \mathbb{R})}^{p}\right),
\end{aligned}
$$

where we also used the fact $\left\|Q^{\frac{1}{2}}\right\|_{\mathcal{L}(\dot{H})} \leq\left\|Q^{\frac{1}{2}}\right\|_{\mathcal{L}_{2}}<\infty$. Taking $\varepsilon>0$ small enough in (3.21), we conclude that

$$
\left\|\sup _{s \in[0, T]} J\left(X_{h}(s)\right)\right\|_{L^{p}(\Omega ; \mathbb{R})}^{p}+\left\|\int_{0}^{T}\left|A_{h} X_{h}(s)+P_{h} F\left(X_{h}(s)\right)\right|_{1}^{2} \mathrm{~d} s\right\|_{L^{p}(\Omega ; \mathbb{R})}^{p}<\infty .
$$

It remains to bound $\left\|A_{h} X_{h}(t)\right\|_{L^{p}(\Omega ; \dot{H})}$. From the definition of the Lyapunov functional $J(\cdot)$ and noting $\Phi(s)=\frac{1}{4}\left(s^{2}-1\right)^{2}$, one can deduce that

$$
|v|_{1}^{2} \leq 2 J(v), \quad \forall v \in \dot{H}^{1},
$$

which leads to

$$
\sup _{s \in[0, T]}\left\|X_{h}(s)\right\|_{L^{p}\left(\Omega ; \dot{H}^{1}\right)} \leq C\left\|\sup _{s \in[0, T]}\left[J\left(X_{h}(s)\right)\right]^{\frac{p}{2}}\right\|_{L^{1}(\Omega ; \mathbb{R})}^{\frac{1}{p}}<\infty .
$$

Using (2.19) shows

$$
\sup _{s \in[0, T]}\left\|F\left(X_{h}(s)\right)\right\|_{L^{p}(\Omega ; H)} \leq C\left(\sup _{s \in[0, T]}\left\|X_{h}(s)\right\|_{L^{p}(\Omega ; \dot{H})}+\sup _{s \in[0, T]}\left\|X_{h}(s)\right\|_{L^{3 p}\left(\Omega ; \dot{H}^{1}\right)}^{3}\right)<\infty .
$$

With the above estimate, one can follow the same lines of the proof of (2.23) to show, for $\delta_{0} \in\left(\frac{3}{2}, 2\right)$,

$$
\begin{aligned}
\sup _{s \in[0, T]}\left\|A_{h}^{\frac{\delta_{0}}{2}} X_{h}(s)\right\|_{L^{p}(\Omega ; \dot{H})} & \leq C\left(\left\|A_{h}^{\frac{\delta_{0}}{2}} P_{h} X_{0}\right\|_{L^{p}(\Omega ; \dot{H})}+\sup _{s \in[0, T]}\left\|F\left(X_{h}(s)\right)\right\|_{L^{p}(\Omega ; H)}+\left\|Q^{\frac{1}{2}}\right\|_{\mathcal{L}_{2}}\right) \\
& \leq C\left(\left\|X_{0}\right\|_{L^{p}\left(\Omega ; \dot{H}^{2}\right)}+1\right)<\infty,
\end{aligned}
$$

where we also used (3.10) in the second step. Similarly to (2.24) in the previous proof, one can show

$$
\left\|X_{h}(t)-X_{h}(s)\right\|_{L^{p}(\Omega ; \dot{H})} \leq C|t-s|^{\frac{\delta_{0}}{4}}, \quad \delta_{0} \in\left(\frac{3}{2}, 2\right),
$$

which together with (2.21) and (3.11) yields,

$$
\begin{aligned}
\left\|A_{h}^{-\frac{1}{2}} P_{h} P\left(F\left(X_{h}(s)\right)-F\left(X_{h}(t)\right)\right)\right\|_{L^{p}(\Omega ; \dot{H})} & \leq C\left\|A^{-\frac{1}{2}} P\left(F\left(X_{h}(s)\right)-F\left(X_{h}(t)\right)\right)\right\|_{L^{p}(\Omega ; \dot{H})} \\
& \left.\leq C\left\|F\left(X_{h}(s)\right)-F\left(X_{h}(t)\right)\right\|_{L^{p}\left(\Omega ; L_{\frac{6}{5}}\right.}\right) \\
& \leq C\left\|X_{h}(s)-X_{h}(t)\right\|_{L^{2 p}(\Omega ; \dot{H})}\left(1+\sup _{s \in[0, T]}\left\|X_{h}(s)\right\|_{L^{4 p}\left(\Omega ; L_{6}\right)}^{2}\right) \\
& \leq C|t-s|^{\frac{\delta_{0}}{4}}, \quad \delta_{0} \in\left(\frac{3}{2}, 2\right) .
\end{aligned}
$$


Combining this with (3.10), (3.24), (3.18), (3.19) and (3.16) with $\mu=0, \frac{5}{2}$ gives, for $\delta_{0} \in\left(\frac{3}{2}, 2\right)$,

$$
\begin{aligned}
\left\|A_{h} X_{h}(t)\right\|_{L^{p}(\Omega ; \dot{H})} \leq & \left\|A_{h} E_{h}(t) P_{h} X_{0}\right\|_{L^{p}(\Omega ; \dot{H})}+\int_{0}^{t}\left\|E_{h}(t-s) A_{h}^{2} P_{h}\left(F\left(X_{h}(s)\right)-F\left(X_{h}(t)\right)\right)\right\|_{L^{p}(\Omega ; \dot{H})} \mathrm{d} s \\
& +\left\|\int_{0}^{t} E_{h}(t-s) A_{h}^{2} P_{h} F\left(X_{h}(t)\right) \mathrm{d} s\right\|_{L^{p}(\Omega ; \dot{H})}+C\left\|\int_{0}^{t} A_{h} E_{h}(t-s) P_{h} \mathrm{~d} W(s)\right\|_{L^{p}(\Omega ; \dot{H})} \\
\leq & C\left\|A_{h} P_{h} X_{0}\right\|_{L^{p}(\Omega ; \dot{H})}+C \int_{0}^{t}(t-s)^{-\frac{5}{4}}\left\|A_{h}^{-\frac{1}{2}} P_{h}\left(F\left(X_{h}(s)\right)-F\left(X_{h}(t)\right)\right)\right\|_{L^{p}(\Omega ; \dot{H})} \mathrm{d} s \\
& +C\left\|F\left(X_{h}(t)\right)\right\|_{L^{p}(\Omega ; H)}+C\left(\int_{0}^{t}\left\|A_{h} E_{h}(t-s) P_{h} Q^{\frac{1}{2}}\right\|_{\mathcal{L}_{2}}^{2} \mathrm{~d} s\right)^{\frac{1}{2}} \\
\leq & C\left(\left\|X_{0}\right\|_{L^{p}\left(\Omega ; \dot{H}^{2}\right)}+\int_{0}^{t}(t-s)^{\frac{\delta_{0}-5}{4}} \mathrm{~d} s+\sup _{s \in[0, T]}\left\|F\left(X_{h}(s)\right)\right\|_{L^{p}(\Omega ; H)}+\left\|Q^{\frac{1}{2}}\right\|_{\mathcal{L}_{2}}\right)<\infty,
\end{aligned}
$$

as required, where the Burkholder-Davis-Gundy inequality was also used.

4. Strong convergence rates of the FEM semi-discretization. The target of this part is to derive error estimates for the semi-discrete finite element approximation of the stochastic problem (1.2). The convergence analysis heavily relies on the moment bounds obtained in the previous section and error estimates for the corresponding deterministic error operators as shown below.

Define two error operators $\Psi_{h}$ and $\Phi_{h}$ for the semi-discrete approximation as follows,

$$
\Psi_{h}(t):=E(t)-E_{h}(t) P_{h} \quad \text { and } \quad \Phi_{h}(t):=A E(t)-A_{h} E_{h}(t) P_{h}, t \in[0, T] .
$$

It is easy to check that

$$
\Psi_{h}(t) v=\Psi_{h}(t) P v, \quad \Phi_{h}(t) v=\Phi_{h}(t) P v, \quad \forall v \in H,
$$

since the constant eigenmodes are cancelled. We present in the following lemma some deterministic semi-discrete error estimates for the above two error operators.

Lemma 4.1. Under Assumptions 2.1, 3.1, the following estimates for $\Psi_{h}$ and $\Phi_{h}$ hold,

$$
\begin{aligned}
\left\|\Psi_{h}(t) v\right\| & \leq C h^{\beta}|v|_{\beta}, \quad \forall v \in \dot{H}^{\beta}, \beta \in[1, r], \\
\left\|\Phi_{h}(t) v\right\| & \leq C h^{\alpha} t^{-1}|v|_{\alpha-2}, \quad \forall v \in \dot{H}^{\alpha-2}, \alpha \in[1, r], \\
\left(\int_{0}^{t}\left\|\Psi_{h}(s) v\right\|^{2} \mathrm{~d} s\right)^{\frac{1}{2}} & \leq C h^{\nu}|\ln h \| v|_{\nu-2}, \quad \forall v \in \dot{H}^{\nu-2}, \nu \in[1, r], \\
\left(\int_{0}^{t}\left\|\Phi_{h}(s) v\right\|^{2} \mathrm{~d} s\right)^{\frac{1}{2}} & \leq C h^{\mu}|\ln h \| v|_{\mu}, \quad \forall v \in \dot{H}^{\mu}, \mu \in[0, r], \\
\left\|\int_{0}^{t} \Phi_{h}(s) v \mathrm{~d} s\right\| & \leq C h^{\varrho}|v|_{\varrho-2}, \quad \forall v \in \dot{H}^{\varrho-2}, \varrho \in[1, r] .
\end{aligned}
$$

Proof of Lemma 4.1. The estimates (4.3) and (4.5) are shown in [30, Theorem 2.1]. Taking (3.6) into account, we can make a slight modification of the proof of [19, (5.6)] in the case $\delta=0$ to prove (4.4). In order to show (4.6), we rely on a simple interpolation between the cases $\mu=0$ and $\mu=r$. The case $\mu=0$ immediately follows from (2.6) with $\varrho=1$ and (3.19). For the case $\mu=r$, we use (3.19), (3.5), (4.1), (3.6) with $i=0, \beta=r$ and (4.5) with $\nu=r$ to deduce

$$
\begin{aligned}
\left(\int_{0}^{t}\left\|\Phi_{h}(s) v\right\|^{2} \mathrm{~d} s\right)^{\frac{1}{2}} & \leq\left(\int_{0}^{t}\left\|\left(E(s)-E_{h}(s) P_{h}\right) A v\right\|^{2} \mathrm{~d} s\right)^{\frac{1}{2}}+\left(\int_{0}^{t}\left\|A_{h} E_{h}(s) P_{h}\left(I-R_{h}\right) v\right\|^{2} \mathrm{~d} s\right)^{\frac{1}{2}} \\
& \leq C h^{r}\left|\operatorname { l n } h \| v | _ { r } + C \| ( R _ { h } - I ) v \left\|\leq C h^{r}|\ln h \| v|_{r} .\right.\right.
\end{aligned}
$$


Finally, an interpolation argument concludes the proof of (4.6). Similarly as before, we use (3.5) to split the term $\left\|\int_{0}^{t} \Phi_{h}(s) v \mathrm{~d} s\right\|$ into two parts:

$$
\begin{aligned}
\left\|\int_{0}^{t} \Phi_{h}(s) v \mathrm{~d} s\right\| & =\left\|\int_{0}^{t}\left(A^{2} E(s) A^{-1}-A_{h}^{2} E_{h}(s) R_{h} A^{-1}\right) v \mathrm{~d} s\right\| \\
& \leq\left\|\int_{0}^{t}\left(A^{2} E(s)-A_{h}^{2} E_{h}(s) P_{h}\right) A^{-1} v \mathrm{~d} s\right\|+\left\|\int_{0}^{t} A_{h}^{2} E_{h}(s) P_{h}\left(R_{h}-I\right) A^{-1} v \mathrm{~d} s\right\| \\
& \leq\left\|\int_{0}^{t} \Psi_{h}^{\prime}(s) A^{-1} v \mathrm{~d} s\right\|+\left\|\int_{0}^{t} E_{h}^{\prime}(s) P_{h}\left(R_{h}-I\right) A^{-1} v \mathrm{~d} s\right\| .
\end{aligned}
$$

For the first term, we use the fundamental theorem of calculus, (3.6) with $i=0, \beta=\varrho$ and (4.3) with $\beta=\varrho$ to show, for $\varrho \in[1, r]$,

$$
\left\|\int_{0}^{t} \Psi_{h}^{\prime}(s) A^{-1} v \mathrm{~d} s\right\|=\left\|\left(\Psi_{h}(t)-\Psi_{h}(0)\right) A^{-1} v\right\| \leq\left\|\Psi_{h}(t) A^{-1} v\right\|+\left\|\left(I-P_{h}\right) A^{-1} v\right\| \leq C h^{\varrho}|v|_{\varrho-2} .
$$

Similarly, we combine the boundness of $E_{h}(s) P_{h}$ in $\dot{H}$ with (3.6) to yield

$$
\left\|\int_{0}^{t} E_{h}^{\prime}(s) P_{h}\left(R_{h}-I\right) A^{-1} v \mathrm{~d} s\right\|=\left\|\left(E_{h}(t)-I\right) P_{h}\left(R_{h}-I\right) A^{-1} v\right\| \leq C h^{\varrho}|v|_{\varrho-2},
$$

which together with (4.9) and (4.8) implies (4.7). This finishes the proof of this lemma.

At the moment, we are well-prepared to prove the main result of this section.

TheOREM 4.2. Let $X(t)$ be the weak solution of (1.2) and let $X_{h}(t)$ be the solution of (3.14). Also, let Assumptions 2.1]2.4 be valid for some $\gamma \in[3,4]$ and let Assumption 3.1 be fulfilled with $r \in\{2,3,4\}$ for $d=1$ and $r=2$ for $d \in\{2,3\}$. Then for any $t \in[0, T]$ and $p \in[1, \infty)$ it holds,

$$
\left\|X(t)-X_{h}(t)\right\|_{L^{p}(\Omega ; \dot{H})} \leq C h^{\kappa}|\ln h|, \quad \text { with } \quad \kappa=\min \{\gamma, r\} .
$$

Moreover, the discrepancy between the "chemical potential" $Y(t):=A X(t)+P F(X(t))$ and its approximation $Y_{h}(t):=A_{h} X_{h}(t)+P_{h} P F\left(X_{h}(t)\right)$ is measured as follows, for any $t \in(0, T]$ and $p \in[1, \infty)$,

$$
\left\|Y(t)-Y_{h}(t)\right\|_{L^{p}(\Omega ; \dot{H})} \leq C\left(1+t^{-1}\right) h^{\iota}|\ln h|, \quad \text { with } \quad \iota=\min \{\gamma-2, r-1\} .
$$

Proof of Theorem 4.2. Since $A$ does not commute with $P_{h}$, the usual arguments splitting the error $X(t)-X_{h}(t)$ into $\left(I-P_{h}\right) X(t)$ and $P_{h} X(t)-X_{h}(t)$ do not work here. To prove this theorem, we propose a different approach and introduce a new auxiliary problem:

$$
\mathrm{d} \widetilde{X}_{h}(t)+A_{h}\left(A_{h} \widetilde{X}_{h}(t)+P_{h} F(X(t))\right) \mathrm{d} t=P_{h} \mathrm{~d} W(t), X_{h}(0)=P_{h} X_{0},
$$

whose unique solution can be written as, in the mild form,

$$
\widetilde{X}_{h}(t)=E_{h}(t) P_{h} X_{0}-\int_{0}^{t} E_{h}(t-s) A_{h} P_{h} F(X(s)) \mathrm{d} s+\int_{0}^{t} E_{h}(t-s) P_{h} \mathrm{~d} W(s) .
$$

Now, we separate the considered error term $\left\|X(t)-X_{h}(t)\right\|_{L^{p}(\Omega ; \dot{H})}$ as follows,

$$
\left\|X(t)-X_{h}(t)\right\|_{L^{p}(\Omega ; \dot{H})} \leq\left\|X(t)-\widetilde{X}_{h}(t)\right\|_{L^{p}(\Omega ; \dot{H})}+\left\|\widetilde{X}_{h}(t)-X_{h}(t)\right\|_{L^{p}(\Omega ; \dot{H})} .
$$


Recall that a similar error decomposition was done in [27, (5.18)]. The first error term can be treated in a standard way. Subtracting (4.14) from (1.4) yields

$$
\begin{aligned}
\left\|X(t)-\widetilde{X}_{h}(t)\right\|_{L^{p}(\Omega ; H)} \leq & \left\|\Psi_{h}(t) X_{0}\right\|_{L^{p}(\Omega ; H)}+\left\|\int_{0}^{t} \Phi_{h}(t-s) F(X(s)) \mathrm{d} s\right\|_{L^{p}(\Omega ; H)} \\
& +\left\|\int_{0}^{t} \Psi_{h}(t-s) \mathrm{d} W(s)\right\|_{L^{p}(\Omega ; H)} \\
= & : I_{1}+I_{2}+I_{3},
\end{aligned}
$$

where the two error operators $\Psi_{h}$ and $\Phi_{h}$ are defined by (4.1). In what follows we treat $I_{1}, I_{2}$ and $I_{3}$, separately. At first, we utilize (4.3) with $\beta=\kappa$ to derive

$$
I_{1} \leq C h^{\kappa}\left\|X_{0}\right\|_{L^{p}\left(\Omega ; \dot{H}^{\kappa}\right)}, \quad \kappa=\min \{\gamma, r\} .
$$

Similarly, employing (2.27), (2.33), (4.2), (4.4) with $\alpha=\kappa$ and (4.7) with $\varrho=\kappa$ yields

$$
\begin{aligned}
I_{2} & \leq\left\|\int_{0}^{t} \Phi_{h}(t-s) P F(X(t)) \mathrm{d} s\right\|_{L^{p}(\Omega ; \dot{H})}+\int_{0}^{t}\left\|\Phi_{h}(t-s) P(F(X(t))-F(X(s)))\right\|_{L^{p}(\Omega ; \dot{H})} \mathrm{d} s \\
& \leq C h^{\kappa}\|P F(X(t))\|_{L^{p}\left(\Omega ; \dot{H}^{\kappa-2}\right)}+C h^{\kappa} \int_{0}^{t}(t-s)^{-1}\|P(F(X(t))-F(X(s)))\|_{L^{p}\left(\Omega ; \dot{H}^{\kappa-2}\right)} \mathrm{d} s \\
& \leq C h^{\kappa} \sup _{t \in[0, T]}\|P F(X(t))\|_{L^{p}\left(\Omega ; \dot{H}^{2}\right)}+C h^{\kappa} \int_{0}^{t}(t-s)^{-\frac{3}{4}} \mathrm{~d} s \\
& \leq C h^{\kappa}, \quad \kappa=\min \{\gamma, r\} .
\end{aligned}
$$

Finally, we use the Burkholder-Davis-Gundy-type inequality and (4.5) with $\nu=\kappa$ to arrive at

$$
I_{3} \leq C_{p}\left(\int_{0}^{t}\left\|\Psi_{h}(t-s) Q^{\frac{1}{2}}\right\|_{\mathcal{L}_{2}}^{2} \mathrm{~d} s\right)^{\frac{1}{2}} \leq C h^{\kappa}|\ln h|\left\|A^{\frac{\kappa-2}{2}} Q^{\frac{1}{2}}\right\|_{\mathcal{L}_{2}} \leq C h^{\kappa}|\ln h|, \quad \kappa=\min \{\gamma, r\} .
$$

Putting the above estimates together yields

$$
\left\|X(t)-\widetilde{X}_{h}(t)\right\|_{L^{p}(\Omega ; \dot{H})} \leq C h^{\kappa}|\ln h|, \quad \kappa=\min \{\gamma, r\} .
$$

Next we turn our attention to the error $\widetilde{e}_{h}(t):=\widetilde{X}_{h}(t)-X_{h}(t)$, which satisfies

$$
\mathrm{d} \widetilde{e}_{h}(t)+A_{h}^{2} \widetilde{e}_{h}(t) \mathrm{d} t=A_{h} P_{h}\left(F(X(t))-F\left(X_{h}(t)\right)\right) \mathrm{d} t, \quad \widetilde{e}_{h}(0)=0 .
$$

Multiplying both sides of (4.19) by $A_{h}^{-1} \widetilde{e}_{h}$, using (2.10), (3.12), (2.19) and recalling the fact $\left\|\widetilde{e}_{h}\right\|^{2} \leq$ $\left|\widetilde{e}_{h}\right|_{1}\left|\widetilde{e}_{h}\right|_{-1, h}$ one obtains

$$
\begin{aligned}
& \frac{1}{2} \frac{\mathrm{d}}{\mathrm{d} s}\left|\widetilde{e}_{h}(s)\right|_{-1, h}^{2}+\left|\widetilde{e}_{h}(s)\right|_{1}^{2}=\left(F\left(\widetilde{X}_{h}(s)\right)-F\left(X_{h}(s)\right), \widetilde{e}_{h}(s)\right)+\left(F(X(s))-F\left(\widetilde{X}_{h}(s)\right), \widetilde{e}_{h}(s)\right) \\
& \quad \leq \frac{3}{2}\left\|\widetilde{e}_{h}(s)\right\|^{2}+\frac{1}{2}\left\|F(X(s))-F\left(\widetilde{X}_{h}(s)\right)\right\|^{2} \\
& \quad \leq \frac{3}{2}\left|\widetilde{e}_{h}(s)\right|_{1}\left|\widetilde{e}_{h}(s)\right|_{-1, h}+C\left\|X(s)-\widetilde{X}_{h}(s)\right\|^{2}\left(1+\left\|\widetilde{X}_{h}(s)\right\|_{V}^{4}+\|X(s)\|_{V}^{4}\right) \\
& \quad \leq \frac{1}{2}\left|\widetilde{e}_{h}(s)\right|_{1}^{2}+\frac{9}{8}\left|\widetilde{e}_{h}(s)\right|_{-1, h}^{2}+C\left\|X(s)-\widetilde{X}_{h}(s)\right\|^{2}\left(1+\left\|A_{h} \widetilde{X}_{h}(s)\right\|^{4}+|X(s)|_{2}^{4}\right) .
\end{aligned}
$$

Integrating over $[0, t]$ and then using Gronwall's inequality one can arrive at

$$
\left|\widetilde{e}_{h}(t)\right|_{-1, h}^{2}+\int_{0}^{t}\left|\widetilde{e}_{h}(s)\right|_{1}^{2} \mathrm{~d} s \leq C \int_{0}^{t}\left\|X(s)-\widetilde{X}_{h}(s)\right\|^{2}\left(1+\left\|A_{h} \widetilde{X}_{h}(s)\right\|^{4}+|X(s)|_{2}^{4}\right) \mathrm{d} s .
$$


In view of (2.27), (3.10), (3.16), (3.19) and the Burkholder-Davis-Gundy inequality, we acquire that

$$
\begin{aligned}
\left\|A_{h} \widetilde{X}_{h}(t)\right\|_{L^{p}(\Omega ; \dot{H})} \leq & \left\|A_{h} E_{h}(t) P_{h} X_{0}\right\|_{L^{p}(\Omega ; \dot{H})}+\int_{0}^{t}\left\|E_{h}(t-s) A_{h}^{2} P_{h} F(X(s))\right\|_{L^{p}(\Omega ; \dot{H})} \mathrm{d} s \\
& +C\left(\int_{0}^{t}\left\|A_{h} E_{h}(t-s) P_{h} Q^{\frac{1}{2}}\right\|_{\mathcal{L}_{2}}^{2} \mathrm{~d} s\right)^{\frac{1}{2}} \\
\leq & \left\|A_{h} P_{h} X_{0}\right\|_{L^{p}(\Omega ; \dot{H})}+C \int_{0}^{t}(t-s)^{-\frac{1}{2}}\left\|A_{h} P_{h} F(X(s))\right\|_{L^{p}(\Omega ; \dot{H})} \mathrm{d} s+C\left\|Q^{\frac{1}{2}}\right\|_{\mathcal{L}_{2}} \\
\leq & C\left(\left\|X_{0}\right\|_{L^{p}\left(\Omega ; \dot{H}^{2}\right)}+\int_{0}^{t}(t-s)^{-\frac{1}{2}} \mathrm{~d} s \sup _{s \in[0, T]}\|P F(X(s))\|_{L^{p}\left(\Omega ; \dot{H}^{2}\right)}+\left\|Q^{\frac{1}{2}}\right\|_{\mathcal{L}_{2}}\right)<\infty .
\end{aligned}
$$

By employing (4.18), (4.22) and (2.17), we derive from (4.21) that

$$
\begin{aligned}
& \left\|\int_{0}^{t}\left|\widetilde{e}_{h}(s)\right|_{1}^{2} \mathrm{~d} s\right\|_{L^{p}(\Omega ; \mathbb{R})} \leq C\left(\int_{0}^{t}\|\| X(s)-\widetilde{X}_{h}(s)\left\|^{2}\left(1+\left\|A_{h} \widetilde{X}_{h}(s)\right\|^{4}+|X(s)|_{2}^{4}\right)\right\|_{L^{p}(\Omega ; \mathbb{R})} \mathrm{d} s\right) \\
& \quad \leq C\left(\int_{0}^{t}\left\|X(s)-\widetilde{X}_{h}(s)\right\|_{L^{4 p}(\Omega ; \dot{H})}^{4} \mathrm{~d} s\right)^{\frac{1}{2}}\left(\int_{0}^{t}\left(1+\left\|A_{h} \widetilde{X}_{h}(s)\right\|_{L^{8 p}(\Omega ; \dot{H})}^{8}+\|X(s)\|_{L^{8 p}\left(\Omega ; \dot{H}^{2}\right)}^{8}\right) \mathrm{d} s\right)^{\frac{1}{2}} \\
& \quad \leq C h^{2 \kappa}|\ln h|^{2}, \quad \kappa=\min \{\gamma, r\} .
\end{aligned}
$$

Equipped with this, we are ready to bound $\left\|\widetilde{e}_{h}(t)\right\|_{L^{p}(\Omega ; \dot{H})}$, which can be decomposed by the following two terms:

$$
\begin{aligned}
\left\|\widetilde{e}_{h}(t)\right\|_{L^{p}(\Omega ; \dot{H})}= & \left\|\int_{0}^{t} E_{h}(t-s) A_{h} P_{h} P\left(F(X(s))-F\left(X_{h}(s)\right)\right) \mathrm{d} s\right\|_{L^{p}(\Omega ; \dot{H})} \\
\leq & \int_{0}^{t}\left\|E_{h}(t-s) A_{h} P_{h} P\left(F(X(s))-F\left(\widetilde{X}_{h}(s)\right)\right)\right\|_{L^{p}(\Omega ; \dot{H})} \mathrm{d} s \\
& +\left\|\int_{0}^{t} E_{h}(t-s) A_{h} P_{h} P\left(F\left(\widetilde{X}_{h}(s)\right)-F\left(X_{h}(s)\right)\right) \mathrm{d} s\right\|_{L^{p}(\Omega ; \dot{H})} \\
= & : J_{1}+J_{2} .
\end{aligned}
$$

Following the same arguments as the proof of (4.23), one can show

$$
\begin{aligned}
& J_{1} \leq C \int_{0}^{t}(t-s)^{-\frac{1}{2}}\left\|F\left(\widetilde{X}_{h}(s)\right)-F(X(s))\right\|_{L^{p}(\Omega ; H)} \mathrm{d} s \\
& \leq C \int_{0}^{t}(t-s)^{-\frac{1}{2}}\left\|X(s)-\widetilde{X}_{h}(s)\right\|_{L^{2 p}(\Omega ; \dot{H})}\left(1+\left\|A_{h} \widetilde{X}_{h}(s)\right\|_{L^{4 p}(\Omega ; \dot{H})}^{2}+\|X(s)\|_{L^{4 p}\left(\Omega ; \dot{H}^{2}\right)}^{2}\right) \mathrm{d} s \\
& \leq C h^{\kappa}|\ln h|, \quad \kappa=\min \{\gamma, r\} .
\end{aligned}
$$

Before handling the term $J_{2}$, we first adapt similar arguments used in the proof of (2.34) and also use (3.12) to get

$$
\begin{aligned}
\left\|A_{h}^{\frac{1}{2}} P_{h} P\left(F\left(X_{h}(s)\right)-F\left(\widetilde{X}_{h}(s)\right)\right)\right\| & \leq\left\|A^{\frac{1}{2}} P\left(F\left(X_{h}(s)\right)-F\left(\widetilde{X}_{h}(s)\right)\right)\right\| \\
& \leq C\left|\widetilde{e}_{h}(s)\right|_{1}\left(1+\left\|A_{h} X_{h}(s)\right\|^{2}+\left\|A_{h} \widetilde{X}_{h}(s)\right\|^{2}\right) .
\end{aligned}
$$


This combined with (4.23), (4.22) and (3.20) yields

$$
\begin{aligned}
J_{2} & \leq\left\|\int_{0}^{t}(t-s)^{-\frac{1}{4}}\right\| A_{h}^{\frac{1}{2}} P_{h}\left(F\left(X_{h}(s)\right)-F\left(\widetilde{X}_{h}(s)\right)\right)\|\mathrm{d} s\|_{L^{p}(\Omega ; \mathbb{R})} \\
& \leq C\left\|\int_{0}^{t}(t-s)^{-\frac{1}{4}}\left|\widetilde{e}_{h}(s)\right|_{1}\left(1+\left\|A_{h} \widetilde{X}_{h}(s)\right\|^{2}+\left\|A_{h} X_{h}(s)\right\|^{2}\right) \mathrm{d} s\right\|_{L^{p}(\Omega ; \mathbb{R})} \\
& \leq C\left\|\left(\int_{0}^{t}\left|\widetilde{e}_{h}(s)\right|_{1}^{2} \mathrm{~d} s\right)^{\frac{1}{2}}\left(\int_{0}^{t}(t-s)^{-\frac{1}{2}}\left(1+\left\|A_{h} \widetilde{X}_{h}(s)\right\|^{2}+\left\|A_{h} X_{h}(s)\right\|^{2}\right)^{2} \mathrm{~d} s\right)^{\frac{1}{2}}\right\|_{L^{p}(\Omega ; \mathbb{R})} \\
& \leq C\left\|\int_{0}^{t}\left|\widetilde{e}_{h}(s)\right|_{1}^{2} \mathrm{~d} s\right\|_{L^{p}(\Omega ; \mathbb{R})}^{\frac{1}{2}}\left\|\int_{0}^{t}(t-s)^{-\frac{1}{2}}\left(1+\left\|A_{h} \widetilde{X}_{h}(s)\right\|^{2}+\left\|A_{h} X_{h}(s)\right\|^{2}\right)^{2} \mathrm{~d} s\right\|_{L^{p}(\Omega ; \mathbb{R})}^{\frac{1}{2}} \\
& \leq C h^{\kappa}|\ln h|, \quad \kappa=\min \{\gamma, r\} .
\end{aligned}
$$

Therefore, gathering the estimates of $J_{1}$ and $J_{2}$ together gives

$$
\left\|\widetilde{X}_{h}(t)-X_{h}(t)\right\|_{L^{p}(\Omega ; \dot{H})} \leq C h^{\kappa}|\ln h|
$$

which combined with (4.18) validates (4.11).

We are now in the position to verify (4.12). Similarly as before, we bound two terms $\| Y(t)-$ $\widetilde{Y}_{h}(t) \|_{L^{p}(\Omega ; \dot{H})}$ and $\left\|\widetilde{Y}_{h}(t)-Y_{h}(t)\right\|_{L^{p}(\Omega ; \dot{H})}$, where $\widetilde{Y}_{h}(t):=A_{h} \widetilde{X}_{h}(t)+P_{h} P F(X(t))$. By (1.4) and (4.14), the error $\left\|Y(t)-\widetilde{Y}_{h}(t)\right\|_{L^{p}(\Omega ; \dot{H})}$ can be decomposed as follows:

$$
\begin{aligned}
\left\|Y(t)-\widetilde{Y}_{h}(t)\right\|_{L^{p}(\Omega ; \dot{H})} \leq & \underbrace{\left\|\left(I-P_{h}\right) P F(X(t))\right\|_{L^{p}(\Omega ; \dot{H})}+\left\|\left(A E(t)-A_{h} E_{h}(t) P_{h}\right) X_{0}\right\|_{L^{p}(\Omega ; \dot{H})}}_{L_{1}} \\
& +\underbrace{\left\|\int_{0}^{t}\left(A^{2} E(t-s)-A_{h}^{2} E_{h}(t-s) P_{h}\right) P F(X(s)) \mathrm{d} s\right\|_{L^{p}(\Omega ; \dot{H})}}_{L_{2}} \\
& +\underbrace{\left\|\int_{0}^{t}\left(A E(t-s)-A_{h} E_{h}(t-s) P_{h}\right) \mathrm{d} W(s)\right\|_{L^{p}(\Omega ; \dot{H})}}_{L_{3}} \cdot
\end{aligned}
$$

Using (3.6), (2.27) and (4.4) with $\alpha=2$ gives

$$
L_{1} \leq C h^{2} \sup _{s \in[0, T]}\|P F(X(s))\|_{L^{p}\left(\Omega ; \dot{H}^{2}\right)}+C h^{2} t^{-1}\left\|X_{0}\right\|_{L^{p}(\Omega ; \dot{H})} \leq C h^{2}\left(1+t^{-1}\right) .
$$

To deal with the term $L_{2}$, we use (3.5) and the definition of the operator $\Phi_{h}(t)$ in (4.1) to get

$$
\begin{aligned}
L_{2} \leq & \left\|\int_{0}^{t} \Phi_{h}(t-s) A P F(X(s)) \mathrm{d} s\right\|_{L^{p}(\Omega ; \dot{H})} \\
& +\left\|\int_{0}^{t} A_{h}^{2} E_{h}(t-s) P_{h}\left(R_{h}-I\right) P F(X(s)) \mathrm{d} s\right\|_{L^{p}(\Omega ; \dot{H})} \\
= & : L_{21}+L_{22} .
\end{aligned}
$$


Owing to (2.27), (2.33) with $\beta=2$, (4.7) with $\varrho=2$ and (4.4) with $\alpha=2$, we infer

$$
\begin{aligned}
L_{21} & \leq\left\|\int_{0}^{t} \Phi_{h}(t-s) A P F(X(t)) \mathrm{d} s\right\|_{L^{p}(\Omega ; \dot{H})}+\int_{0}^{t}\left\|\Phi_{h}(t-s) A P(F(X(s))-F(X(t)))\right\|_{L^{p}(\Omega ; \dot{H})} \mathrm{d} s \\
& \leq C h^{2}\|P F(X(t))\|_{L^{p}\left(\Omega ; \dot{H}^{2}\right)}+C h^{2} \int_{0}^{t}(t-s)^{-1}\|P(F(X(s))-F(X(t)))\|_{L^{p}\left(\Omega ; \dot{H}^{2}\right)} \mathrm{d} s \\
& \leq C h^{2} \sup _{s \in[0, T]}\|P F(X(s))\|_{L^{p}\left(\Omega ; \dot{H}^{2}\right)}+C h^{2} \int_{0}^{t}(t-s)^{-1}(t-s)^{\frac{1}{4}} \mathrm{~d} s \\
& \leq C h^{2} .
\end{aligned}
$$

Likewise, we use (2.27), (3.18), (2.33) with $\beta=2$ and (3.16) with $\mu=2$ to derive

$$
\begin{aligned}
L_{22} \leq & \int_{0}^{t}\left\|A_{h}^{2} E_{h}(t-s) P_{h}\left(R_{h}-I\right) P(F(X(s))-F(X(t)))\right\|_{L^{p}(\Omega ; \dot{H})} \mathrm{d} s \\
& +\left\|\int_{0}^{t} A_{h}^{2} E_{h}(t-s) P_{h}\left(R_{h}-I\right) P F(X(t)) \mathrm{d} s\right\|_{L^{p}(\Omega ; \dot{H})} \\
\leq & C h^{2} \int_{0}^{t}(t-s)^{-1}\|P(F(X(s))-F(X(t)))\|_{L^{p}\left(\Omega ; \dot{H}^{2}\right)} \mathrm{d} s \\
& +C\left\|\left(R_{h}-I\right) P F(X(t))\right\|_{L^{p}(\Omega ; \dot{H})} \\
\leq & C h^{2} \int_{0}^{t}(t-s)^{-1}(t-s)^{\frac{1}{4}} \mathrm{~d} s+C h^{2} \sup _{s \in[0, T]}\|P F(X(s))\|_{L^{p}\left(\Omega ; \dot{H}^{2}\right)} \\
\leq & C h^{2},
\end{aligned}
$$

which together with (4.31) implies

$$
L_{2} \leq C h^{2}
$$

With the aid of the Burkholder-Davis-Gundy inequality, one can use (4.6) with $\mu=\gamma-2$ to deduce

$$
L_{3} \leq C\left(\int_{0}^{t}\left\|\Phi_{h}(t-s) Q^{\frac{1}{2}}\right\|_{\mathcal{L}_{2}}^{2} \mathrm{~d} s\right)^{\frac{1}{2}} \leq C h^{\gamma-2}|\ln h|\left\|A^{\frac{\gamma-2}{2}} Q^{\frac{1}{2}}\right\|_{\mathcal{L}_{2}} \leq C h^{\gamma-2}|\ln h| .
$$

Gathering (4.29), 4.33) and (4.34) together implies

$$
\left\|Y(t)-\widetilde{Y}_{h}(t)\right\|_{L^{p}(\Omega ; \dot{H})} \leq C h^{\gamma-2}|\ln h|\left(1+t^{-1}\right), \quad \forall t \in(0, T]
$$

To bound the error $\left\|\widetilde{Y}_{h}\left(t_{n}\right)-Y_{h}\left(t_{n}\right)\right\|_{L^{p}(\Omega ; \dot{H})}$, we first apply (2.10), (3.12), (2.19), (2.17), (3.20) and (4.11) to achieve

$$
\begin{aligned}
\left\|P\left(F(X(t))-F\left(X_{h}(t)\right)\right)\right\|_{L^{p}(\Omega ; \dot{H})} & \leq\left(1+\|X(t)\|_{L^{4 p}(\Omega ; V)}^{2}+\left\|X_{h}(t)\right\|_{L^{4 p}(\Omega ; V)}^{2}\right)\left\|X(t)-X_{h}(t)\right\|_{L^{2 p}(\Omega ; \dot{H})} \\
& \leq\left(1+\|X(t)\|_{L^{4 p}\left(\Omega ; \dot{H}^{2}\right)}^{2}+\left\|A_{h} X_{h}(t)\right\|_{L^{4 p}(\Omega ; \dot{H})}^{2}\right)\left\|X(t)-X_{h}(t)\right\|_{L^{2 p}(\Omega ; \dot{H})} \\
& \leq C h^{\kappa}|\ln h| .
\end{aligned}
$$


Combining this with the inverse inequality (3.9) enables us to obtain

$$
\begin{aligned}
\left\|\widetilde{Y}_{h}\left(t_{n}\right)-Y_{h}\left(t_{n}\right)\right\|_{L^{p}(\Omega ; \dot{H})} \leq & \int_{0}^{t}\left\|A_{h}^{2} E_{h}(t-s) P_{h} P\left(F(X(s))-F\left(X_{h}(s)\right)\right)\right\|_{L^{p}(\Omega ; \dot{H})} \mathrm{d} s \\
& +\left\|P_{h} P\left(F(X(t))-F\left(X_{h}(t)\right)\right)\right\|_{L^{p}(\Omega ; H)} \\
\leq & C h^{-1} \int_{0}^{t}(t-s)^{-\frac{3}{4}}\left\|F(X(s))-F\left(X_{h}(s)\right)\right\|_{L^{p}(\Omega ; H)} \mathrm{d} s+C h^{\kappa}|\ln h| \\
\leq & C h^{\kappa-1}|\ln h|,
\end{aligned}
$$

which in conjunction with (4.35) implies the desired assertion (4.12).

5. The finite element full-discretization and its moment bounds. In this section, we proceed to look at a finite element full discretization of (1.2) and provide moment bounds of the full discretization. Let $k=T / N, N \in \mathbb{N}$ be a uniform time step-size and $t_{n}=k n, n=1,2, \cdots, N$. We discretize (3.14) in time with a backward Euler scheme and the resulting fully discrete problem is to find $\mathcal{F}_{t_{n}}$-adapted $\dot{V}_{h}$-valued random variable $X_{h}^{n}$ such that

$$
X_{h}^{n}-X_{h}^{n-1}+k A_{h}^{2} X_{h}^{n}+k A_{h} P_{h} F\left(X_{h}^{n}\right)=P_{h} \Delta W_{n}, X_{h}^{0}=P_{h} X_{0}, \quad n=1,2, \cdots, N,
$$

where $\Delta W_{n}:=W\left(t_{n}\right)-W\left(t_{n-1}\right)$. Noting that the above implicit scheme works on the finite dimensional space $\dot{V}_{h}$ and that the mapping $A_{h}^{2}+k A_{h} P_{h} F(\cdot)$ obeys a kind of monotonicity condition in the Hilbert space $\left(\dot{V}_{h},(\cdot, \cdot)_{-1, h}\right)$, one can check that the implicit scheme (5.1) is well-posed in $\dot{V}_{h}$. After introducing a family of operators $\left\{E_{k, h}^{n}\right\}_{n=1}^{N}$ :

$$
E_{k, h}^{n} v_{h}:=\left(I+k A_{h}^{2}\right)^{-n} v_{h}=\sum_{j=0}^{\mathcal{N}_{h}}\left(1+k \lambda_{j, h}^{2}\right)^{-n}\left(v_{h}, e_{j, h}\right) e_{j, h}, \quad \forall v_{h} \in V_{h},
$$

the solution of (5.1), similarly to the semi-discrete case, can be expressed in the following form,

$$
X_{h}^{n}=E_{k, h}^{n} P_{h} X_{0}-k \sum_{j=1}^{n} A_{h} E_{k, h}^{n-j+1} P_{h} P F\left(X_{h}^{j}\right)+\sum_{j=1}^{n} E_{k, h}^{n-j+1} P_{h} \Delta W_{j}, \quad n=0,1,2, \cdots, N .
$$

The next theorem offers a priori moment bounds for the fully-discrete approximations.

TheOREM 5.1. Let $X_{h}^{n}$ be given by (5.1). If Assumptions 2.1 2.4 3.1 are valid, then there exist $k_{0}>0$ such that for all $k \leq k_{0}, h>0$ and $p \geq 1$,

$$
\sup _{1 \leq n \leq N}\left\|A_{h} X_{h}^{n}\right\|_{L^{p}(\Omega ; \dot{H})}+\left\|\sum_{j=1}^{N} k\left|A_{h} X_{h}^{j}+P_{h} F\left(X_{h}^{j}\right)\right|_{1}^{2}\right\|_{L^{p}(\Omega ; \mathbb{R})}<\infty .
$$

To prove it, we first introduce some smooth properties of the operator $E_{k, h}^{n}$. Denoting $r(z):=$ $(1+z)^{-1}$, one can write $E_{k, h}^{n}=r\left(k A_{h}^{2}\right)^{n}$. As shown in [38, Theorem 7.1], there exist two constants $C$ and $c$ such that

$$
\left|r(z)-e^{-z}\right| \leq C z^{2}, \quad \forall z \in[0,1]
$$

and

$$
|r(z)| \leq e^{-c z}, \forall z \in[0,1]
$$


These two inequalities suffice to ensure that, for $n=1,2,3, \cdots$,

$$
\left|r(z)^{n}-e^{-z n}\right| \leq\left|\left(r(z)-e^{-z}\right) \sum_{l=0}^{n-1} r(z)^{n-1-l} e^{-z l}\right| \leq C n z^{2} e^{-c(n-1) z}, \quad \forall z \in[0,1] .
$$

Additionally, we need a temporal discrete analogue of Lemma 3.1 as follows.

Lemma 5.2. Under Assumptions 2.1. 3.1, the following estimates hold for $n=1,2,3, \cdots, N$,

$$
\begin{aligned}
\left\|A_{h}^{\mu} E_{k, h}^{n} P_{h} v\right\| & \leq C t_{n}^{-\frac{\mu}{2}}\|v\|, \quad \forall \mu \in[0,2], v \in \dot{H}, \\
\left\|A_{h}^{-\nu}\left(I-E_{k, h}^{n}\right) P_{h} v\right\| & \leq C t_{n}^{\frac{\nu}{2}}\|v\|, \quad \forall \nu \in[0,2], v \in \dot{H}, \\
\left\|k \sum_{j=1}^{n} A_{h}^{2} E_{k, h}^{j} P_{h} v\right\| & \leq C\|v\|, \quad \forall v \in \dot{H}, \\
\left(k \sum_{j=1}^{n}\left\|A_{h} E_{k, h}^{j} P_{h} v\right\|^{2}\right)^{\frac{1}{2}} & \leq C\|v\|, \quad \forall v \in \dot{H} .
\end{aligned}
$$

Proof of Lemma [5.2. The estimate (5.6) can be found in [19, (2.10)]. And the proof of (5.7) is based on an interpolation argument. Taking $\mu=0$ in (5.6) gives (5.7) for the case $\nu=0$. To show (5.7) for the case $\nu=2$, we expand $P_{h} v$ in terms of $\left\{e_{j, h}\right\}_{j=1}^{\mathcal{N}_{h}}$ to obtain

$$
\begin{aligned}
\left\|A_{h}^{-2}\left(I-E_{k, h}^{n}\right) P_{h} v\right\|^{2} & =\left\|\sum_{j=1}^{\mathcal{N}_{h}} \lambda_{j, h}^{-2}\left(1-r\left(k \lambda_{j, h}^{2}\right)^{n}\right)\left(v, e_{j, h}\right) e_{j, h}\right\|^{2} \\
& =\sum_{j=1}^{\mathcal{N}_{h}} \lambda_{j, h}^{-4}\left(1-r\left(k \lambda_{j, h}^{2}\right)^{n}\right)^{2}\left(v, e_{j, h}\right)^{2}
\end{aligned}
$$

where $\left\{\lambda_{j, h}\right\}_{j=1}^{\mathcal{N}_{h}}$ are the positive eigenvalues of $A_{h}$ with corresponding orthonormal eigenvectors $\left\{e_{j, h}\right\}_{j=1}^{\mathcal{N}_{h}} \subset \dot{V}_{h}$. Since

$$
\left|1-r\left(k \lambda_{j, h}^{2}\right)^{n}\right|=\left|1-\left(1+k \lambda_{j, h}^{2}\right)^{-n}\right| \leq t_{n} \lambda_{j, h}^{2}, \quad j=1,2, \cdots, \mathcal{N}_{h},
$$

one can derive from (5.10) that (5.7) holds for the case $\nu=2$. The intermediate cases follow by an interpolation argument. To show (5.8), we again use Parseval's identity to infer

$$
\left\|k \sum_{j=1}^{n} A_{h}^{2} E_{k, h}^{j} P_{h} v\right\|^{2}=\left\|k \sum_{j=1}^{n} \sum_{i=1}^{\mathcal{N}_{h}} \lambda_{i, h}^{2} r\left(k \lambda_{i, h}^{2}\right)^{j}\left(v, e_{i, h}\right) e_{i, h}\right\|^{2}=\sum_{i=1}^{\mathcal{N}_{h}}\left(k \sum_{j=1}^{n} \lambda_{i, h}^{2} r\left(k \lambda_{i, h}^{2}\right)^{j}\right)^{2}\left(v, e_{i, h}\right)^{2} .
$$

Therefore, (5.8) holds on the condition,

$$
k \sum_{j=1}^{n} \lambda_{i, h}^{2} r\left(k \lambda_{i, h}^{2}\right)^{j} \leq C, \quad i=1,2, \cdots, \mathcal{N}_{h} .
$$

Next, we validate (5.12) by considering two possibilities: either $k \lambda_{i, h}^{2} \leq 1$ or $k \lambda_{i, h}^{2}>1$. For the first possibility that $k \lambda_{i, h}^{2} \leq 1$, we use (5.4) to deduce

$$
k \sum_{j=1}^{n} \lambda_{i, h}^{2} r\left(k \lambda_{i, h}^{2}\right)^{j} \leq k \sum_{j=1}^{n} \lambda_{i, h}^{2} e^{-c t_{j} \lambda_{i, h}^{2}} \leq \int_{0}^{t_{n}} \lambda_{i, h}^{2} e^{-c s \lambda_{i, h}^{2}} \mathrm{~d} s \leq \frac{1}{c}\left(1-e^{-c t_{n} \lambda_{i, h}^{2}}\right) \leq \frac{1}{c} .
$$


For the other possibility that $k \lambda_{i, h}^{2}>1$, we notice that $r\left(k \lambda_{i, h}^{2}\right)<\frac{1}{2}$ and thus

$$
k \sum_{j=1}^{n} \lambda_{i, h}^{2} r\left(k \lambda_{i, h}^{2}\right)^{j} \leq \sum_{j=1}^{n} r\left(k \lambda_{i, h}^{2}\right)^{j-1}<\sum_{j=1}^{n} 2^{-(j-1)} \leq 2 .
$$

As a consequence, we obtain (5.12) and the assertion (5.8) follows. The proof of (5.9) is similar to that of (5.8) and we omit it.

Equipped with the above preparations, we are prepared to show Theorem 5.1

Proof of Theorem [5.1. Following almost the same lines as in the proof of [19, Theorem 4.3], one can arrive at

$$
\begin{aligned}
& \left\|\sup _{1 \leq j \leq N} J\left(X_{h}^{j}\right)\right\|_{L^{p}(\Omega ; \mathbb{R})}^{p}+\left\|\sum_{j=1}^{N} k\left|A_{h} X_{h}^{j}+P_{h} F\left(X_{h}^{j}\right)\right|_{1}^{2}\right\|_{L^{p}(\Omega ; \mathbb{R})}^{p} \\
& \quad \leq C\left(1+\left\|J\left(P_{h} X_{0}\right)\right\|_{L^{p}(\Omega ; \mathbb{R})}^{p}+\left\|P_{h} X_{0}\right\|_{L^{4 p+1}(\Omega ; \dot{H})}^{4 p+1}+\left\|Q^{\frac{1}{2}}\left(A_{h} P_{h} X_{0}+P_{h} F\left(P_{h} X_{0}\right)\right)\right\|_{L^{2 p}(\Omega ; \dot{H})}^{2 p}\right) \\
& \quad<\infty .
\end{aligned}
$$

The boundedness holds because $\left\|J\left(P_{h} X_{0}\right)\right\|_{L^{p}(\Omega ; \mathbb{R})}^{p}+\left\|P_{h} X_{0}\right\|_{L^{4 p+1}(\Omega ; \dot{H})}^{4 p+1}<\infty$ and

$$
\begin{aligned}
& \left\|Q^{\frac{1}{2}}\left(A_{h} P_{h} X_{0}+P_{h} F\left(P_{h} X_{0}\right)\right)\right\|_{L^{2 p}(\Omega ; \dot{H})}^{2 p} \\
& \quad \leq C\left\|Q^{\frac{1}{2}}\right\|_{\mathcal{L}(\dot{H})}^{2 p}\left(\left\|A_{h} P_{h} X_{0}\right\|_{L^{2 p}(\Omega ; \dot{H})}^{2 p}+\left\|P_{h} X_{0}\right\|_{L^{2 p}(\Omega ; \dot{H})}^{2 p}+\left\|P_{h} X_{0}\right\|_{L^{6 p}\left(\Omega ; \dot{H}^{1}\right)}^{6 p}\right) \\
& \quad \leq C\left(1+\left\|X_{0}\right\|_{L^{2 p}\left(\Omega ; \dot{H}^{2}\right)}^{2 p}+\left\|X_{0}\right\|_{L^{6 p}\left(\Omega ; \dot{H}^{1}\right)}^{6 p}\right)<\infty,
\end{aligned}
$$

due to the use of (2.19), (3.7), (3.8) and (3.10). Now it remains to bound the term $\left\|A_{h} X_{h}^{n}\right\|_{L^{p}(\Omega ; \dot{H})}$. Similarly to (3.23), we recall (3.22) and obtain

$$
\sup _{1 \leq j \leq N}\left\|X_{h}^{j}\right\|_{L^{p}\left(\Omega ; \dot{H}^{1}\right)} \leq C\left\|\sup _{1 \leq j \leq N} J\left(X_{h}^{j}\right)^{\frac{p}{2}}\right\|_{L^{1}(\Omega ; \mathbb{R})}^{\frac{1}{p}}<\infty
$$

which can be used to promise

$$
\sup _{1 \leq j \leq N}\left\|F\left(X_{h}^{j}\right)\right\|_{L^{p}(\Omega ; H)} \leq\left(\sup _{1 \leq j \leq N}\left\|X_{h}^{j}\right\|_{L^{p}(\Omega ; \dot{H})}+\sup _{1 \leq j \leq N}\left\|X_{h^{j}}^{j}\right\|_{L^{3 p}\left(\Omega ; \dot{H}^{1}\right)}^{3}\right)<\infty .
$$

With this at hand, one can follow the same lines in the proof of (3.27) to get

$$
\sup _{1 \leq n \leq N}\left\|A_{h} X_{h}^{n}\right\|_{L^{p}(\Omega ; \dot{H})}<\infty
$$

The proof of this theorem is complete.

6. Strong convergence rates of the FEM full-discretization. The goal of this section is to identify strong convergence rates of the fully discrete finite element method (5.1). Similarly to the semi-discrete case, error estimates for the deterministic error operators and the moment bounds of the fully discrete finite element solutions together play a key role in the convergence analysis. To begin with, we define the corresponding error operators

$$
\Psi_{k, h}(t):=E(t)-E_{k, h}^{n} P_{h} \text { and } \Phi_{k, h}(t):=A E(t)-A_{h} E_{k, h}^{n} P_{h}, \quad t \in\left[t_{n-1}, t_{n}\right), n \in\{1,2, \cdots, N\} .
$$


Since the constant eigenmodes are canceled, it is easy to see

$$
\Phi_{h}(t) v=\Phi_{h}(t) P v, \quad v \in H .
$$

The following lemma is a temporal version of Lemma 4.1 which is crucial in the error analysis.

Lemma 6.1. Under Assumptions 2.1, 3.1, the following estimates for $\Psi_{k, h}(t)$ and $\Phi_{k, h}(t)$ hold

$$
\begin{aligned}
\left\|\Psi_{k, h}(t) v\right\| & \leq C\left(h^{\beta}+k^{\frac{\beta}{4}}\right)|v|_{\beta}, \quad \forall \beta \in[1, r], v \in \dot{H}^{\beta}, t \in[0, T], \\
\left\|\Phi_{k, h}(t) v\right\| & \leq C\left(h^{\alpha}+k^{\frac{\alpha}{4}}\right) t^{-1}|v|_{\alpha-2}, \quad \forall \alpha \in[1, r], v \in \dot{H}^{\alpha-2}, t \in(0, T], \\
\left(\int_{0}^{t}\left\|\Psi_{k, h}(s) v\right\|^{2} \mathrm{~d} s\right)^{\frac{1}{2}} & \leq C\left(h^{\nu}|\ln h|+|\ln k| k^{\frac{\nu}{4}}\right)|v|_{\nu-2}, \quad \forall v \in \dot{H}^{\nu-2}, \nu \in[1, r], t \in[0, T], \\
\left(\int_{0}^{t}\left\|\Phi_{k, h}(s) v\right\|^{2} \mathrm{~d} s\right)^{\frac{1}{2}} & \leq C\left(h^{\mu}|\ln h|+k^{\frac{\mu}{4}}|\ln k|\right)|v|_{\mu}, \quad \forall \mu \in[0, r], v \in \dot{H}^{\mu}, t \in[0, T], \\
\left\|\int_{0}^{t} \Phi_{k, h}(s) v \mathrm{~d} s\right\| & \leq C\left(h^{\varrho}+k^{\frac{\varrho}{4}}\right)|v|_{\varrho-2}, \quad \forall \varrho \in[1, r], v \in \dot{H}^{\varrho-2}, t \in[0, T],
\end{aligned}
$$

where $r \in\{2,3,4\}$ for $d=1$ and $r=2$ for $d \in\{2,3\}$, as implied by Assumption [3.1.

The proof of Lemma 6.1 is postponed to Appendix. Equipped with this lemma we are wellprepared to do the error analysis. The next theorem states the main result of this section, concerning strong convergence rates of the FEM full-discretization.

Theorem 6.2. Let $X(t)$ be the weak solution of (1.2) and let $X_{h}^{n}$ be given by (5.1). Let Assumptions 2.1 2.4 be valid for some $\gamma \in[3,4]$ and let Assumption 3.1 be fulfilled with $r \in\{2,3,4\}$ for $d=1$ and $r=2$ for $d \in\{2,3\}$. Then there exist $k_{0}>0$ such that for all $k \leq k_{0}, h>0, p \geq 1$ and $n \in\{0,1, \ldots, N\}$,

$$
\left\|X\left(t_{n}\right)-X_{h}^{n}\right\|_{L^{p}(\Omega ; \dot{H})} \leq C\left(h^{\kappa}|\ln h|+k^{\frac{\kappa}{4}}|\ln k|\right) \quad \text { with } \kappa:=\min \{\gamma, r\} .
$$

Moreover, the discrepancy between the "chemical potential" $Y(t):=A X(t)+P F(X(t))$ and its approximation $Y_{h}^{n}:=A_{h} X_{h}^{n}+P_{h} P F\left(X_{h}^{n}\right)$ is measured as follows, for any $k \leq k_{0}, h>0, p \geq 1$ and $n \in\{1,2, \ldots, N\}$,

$$
\left\|Y\left(t_{n}\right)-Y_{h}^{n}\right\|_{L^{p}(\Omega ; \dot{H})} \leq C\left(1+t_{n}^{-1}\right)\left(h^{\iota}|\ln h|+k^{\frac{\iota}{4}}|\ln k|\right) \quad \text { with } \iota=\min \{\gamma-2, r-1\} .
$$

Proof of Theorem 6.2. Similarly to the semi-discrete case, by introducing the auxiliary problem,

$$
\widetilde{X}_{h}^{n}-\widetilde{X}_{h}^{n-1}+k A_{h}\left(A_{h} \widetilde{X}_{h}^{n}+P_{h} F\left(X\left(t_{n}\right)\right)\right)=P_{h} \Delta W_{n}, \quad \widetilde{X}_{h}^{0}=P_{h} X_{0},
$$

whose solution can be recasted as

$$
\widetilde{X}_{h}^{n}=E_{k, h}^{n} P_{h} X_{0}-k \sum_{j=1}^{n} A_{h} E_{k, h}^{n-j+1} P_{h} F\left(X\left(t_{j}\right)\right)+\sum_{j=1}^{n} E_{k, h}^{n-j+1} P_{h} \Delta W_{j},
$$

we decompose the considered error $\left\|X\left(t_{n}\right)-X_{h}^{n}\right\|_{L^{p}(\Omega ; \dot{H})}$ into two parts:

$$
\left\|X\left(t_{n}\right)-X_{h}^{n}\right\|_{L^{p}(\Omega ; \dot{H})} \leq\left\|X\left(t_{n}\right)-\widetilde{X}_{h}^{n}\right\|_{L^{p}(\Omega ; \dot{H})}+\left\|\widetilde{X}_{h}^{n}-X_{h}^{n}\right\|_{L^{p}(\Omega ; \dot{H})} .
$$

At first we handle the estimate of the first error term. Subtracting (6.11) from (1.4), we split it into 
the following three parts:

$$
\begin{aligned}
\left\|X\left(t_{n}\right)-\widetilde{X}_{h}^{n}\right\|_{L^{p}(\Omega ; \dot{H})} \leq & \left\|\left(E\left(t_{n}\right)-E_{k, h}^{n} P_{h}\right) X_{0}\right\|_{L^{p}(\Omega ; \dot{H})} \\
& +\| \int_{0}^{t_{n}} E\left(t_{n}-s\right) A P F(X(s)) \mathrm{d} s-k \sum_{j=1}^{n} A_{h} E_{k, h}^{n-j+1} P_{h} P F\left(\left(X\left(t_{j}\right)\right) \|_{L^{p}(\Omega ; \dot{H})}\right. \\
& +\left\|\sum_{j=1}^{n} \int_{t_{j-1}}^{t_{j}}\left(E\left(t_{n}-s\right)-E_{k, h}^{n-j+1} P_{h}\right) \mathrm{d} W(s)\right\|_{L^{p}(\Omega ; \dot{H})} \\
= & : \mathbb{I}+\mathbb{J}+\mathbb{K} .
\end{aligned}
$$

The three terms $\mathbb{I}, \mathbb{J}, \mathbb{K}$ will be treated separately. By using (6.3) with $\beta=\kappa$, we estimate the first term $\mathbb{I}$ as follows,

$$
\mathbb{I} \leq C\left(h^{\kappa}+k^{\frac{\kappa}{4}}\right)\left\|X_{0}\right\|_{L^{p}\left(\Omega ; \dot{H}^{\kappa}\right)}, \quad \kappa=\min \{\gamma, r\} .
$$

To bound the term $\mathbb{J}$, we need to decompose it further:

$$
\begin{aligned}
\mathbb{J} \leq & \left\|\sum_{j=1}^{n} \int_{t_{j-1}}^{t_{j}} E\left(t_{n}-s\right) A P\left(F(X(s))-F\left(X\left(t_{j}\right)\right)\right) \mathrm{d} s\right\|_{L^{p}(\Omega ; \dot{H})} \\
& +\left\|\int_{0}^{t_{n}} \Phi_{k, h}\left(t_{n}-s\right) P F\left(X\left(t_{n}\right)\right) \mathrm{d} s\right\|_{L^{p}(\Omega ; \dot{H})} \\
& +\sum_{j=1}^{n} \int_{t_{j-1}}^{t_{j}}\left\|\Phi_{k, h}\left(t_{n}-s\right) P\left(F\left(X\left(t_{j}\right)\right)-F\left(X\left(t_{n}\right)\right)\right)\right\|_{L^{p}(\Omega ; \dot{H})} \mathrm{d} s \\
= & : \mathbb{J}_{1}+\mathbb{J}_{2}+\mathbb{J}_{3} .
\end{aligned}
$$

Noticing that, for $s \in\left[t_{j-1}, t_{j}\right)$,

$$
X\left(t_{j}\right)=E\left(t_{j}-s\right) X(s)-\int_{s}^{t_{j}} E\left(t_{j}-\sigma\right) A P F(X(\sigma)) \mathrm{d} \sigma+\int_{s}^{t_{j}} E\left(t_{j}-\sigma\right) \mathrm{d} W(\sigma),
$$

and using Taylor's formula help us to split $\mathbb{J}_{1}$ into four additional terms:

$$
\begin{aligned}
\mathbb{J}_{1} \leq & \left\|\sum_{j=1}^{n} \int_{t_{j-1}}^{t_{j}} E\left(t_{n}-s\right) A P F^{\prime}(X(s))\left(E\left(t_{j}-s\right)-I\right) X(s) \mathrm{d} s\right\|_{L^{p}(\Omega ; \dot{H})} \\
& +\left\|\sum_{j=1}^{n} \int_{t_{j-1}}^{t_{j}} E\left(t_{n}-s\right) A P F^{\prime}(X(s)) \int_{s}^{t_{j}} E\left(t_{j}-\sigma\right) A P F(X(\sigma)) \mathrm{d} \sigma \mathrm{d} s\right\|_{L^{p}(\Omega ; \dot{H})} \\
& +\left\|\sum_{j=1}^{n} \int_{t_{j-1}}^{t_{j}} E\left(t_{n}-s\right) A P F^{\prime}(X(s)) \int_{s}^{t_{j}} E\left(t_{j}-\sigma\right) \mathrm{d} W(\sigma) \mathrm{d} s\right\|_{L^{p}(\Omega ; \dot{H})} \\
& +\left\|\sum_{j=1}^{n} \int_{t_{j-1}}^{t_{j}} E\left(t_{n}-s\right) A P R_{F}\left(X(s), X\left(t_{j}\right)\right) \mathrm{d} s\right\|_{L^{p}(\Omega ; \dot{H})} \\
=: & \mathbb{J}_{11}+\mathbb{J}_{12}+\mathbb{J}_{13}+\mathbb{J}_{14} .
\end{aligned}
$$

Here the remainder term $R_{F}$ is given by,

$$
R_{F}\left(X(s), X\left(t_{j}\right)\right):=\int_{0}^{1} F^{\prime \prime}\left(X(s)+\lambda\left(X\left(t_{j}\right)-X(s)\right)\right)\left(X\left(t_{j}\right)-X(s), X\left(t_{j}\right)-X(s)\right)(1-\lambda) \mathrm{d} \lambda .
$$


In view of (2.4) with $\nu=\frac{2+\delta_{0}}{2}$, 2.16), (2.17), 2.20) and the Hölder inequality, we derive, for any fixed $\delta_{0} \in\left(\frac{3}{2}, 2\right)$ and $\gamma \in[3,4]$,

$$
\begin{aligned}
\mathbb{J}_{11} & \leq C \sum_{j=1}^{n} \int_{t_{j-1}}^{t_{j}}\left(t_{n}-s\right)^{-\frac{2+\delta_{0}}{4}}\left\|A^{-\frac{\delta_{0}}{2}} P F^{\prime}(X(s))\left(E\left(t_{j}-s\right)-I\right) X(s)\right\|_{L^{p}(\Omega ; \dot{H})} \mathrm{d} s \\
& \leq C \sum_{j=1}^{n} \int_{t_{j-1}}^{t_{j}}\left(t_{n}-s\right)^{-\frac{2+\delta_{0}}{4}}\left\|F^{\prime}(X(s))\left(E\left(t_{j}-s\right)-I\right) X(s)\right\|_{L^{p}\left(\Omega ; L_{1}\right)} \mathrm{d} s \\
& \leq C \sum_{j=1}^{n} \int_{t_{j-1}}^{t_{j}}\left(t_{n}-s\right)^{-\frac{2+\delta_{0}}{4}}\left\|f^{\prime}(X(s))\right\|_{L^{2 p}(\Omega ; H)}\left\|\left(E\left(t_{j}-s\right)-I\right) X(s)\right\|_{L^{2 p}(\Omega ; \dot{H})} \mathrm{d} s \\
& \leq C k^{\frac{\gamma}{4}} \int_{0}^{t_{n}}\left(t_{n}-s\right)^{-\frac{2+\delta_{0}}{4}} \mathrm{~d} s \sup _{s \in[0, T]}\left\|f^{\prime}(X(s))\right\|_{L^{2 p}(\Omega ; H)} \sup _{s \in[0, T]}\|X(s)\|_{L^{2 p}\left(\Omega ; \dot{H}^{\gamma}\right)} \\
& \leq C k^{\frac{\gamma}{4}} .
\end{aligned}
$$

Following similar arguments as in (6.14), we use (2.27) to show that, for any $\delta_{0} \in\left(\frac{3}{2}, 2\right)$,

$$
\begin{aligned}
\mathbb{J}_{12} & \leq \sum_{j=1}^{n} \int_{t_{j-1}}^{t_{j}} \int_{s}^{t_{j}}\left(t_{n}-s\right)^{-\frac{2+\delta_{0}}{4}}\left\|F^{\prime}(X(s)) E\left(t_{i+1}-\sigma\right) A P F(X(\sigma))\right\|_{L^{p}\left(\Omega ; L_{1}\right)} \mathrm{d} \sigma \mathrm{d} s \\
& \leq C \sum_{j=1}^{n} \int_{t_{j-1}}^{t_{j}} \int_{s}^{t_{j}}\left(t_{n}-s\right)^{-\frac{2+\delta_{0}}{4}}\left\|f^{\prime}(X(s))\right\|_{L^{2 p}(\Omega ; H)}\|P F(X(\sigma))\|_{L^{2 p}\left(\Omega ; \dot{H}^{2}\right)} \mathrm{d} \sigma \mathrm{d} s \\
& \leq C k \int_{0}^{t_{n}}\left(t_{n}-s\right)^{-\frac{2+\delta_{0}}{4}} \mathrm{~d} s \sup _{s \in[0, T]}\left\|f^{\prime}(X(s))\right\|_{L^{2 p}(\Omega ; H)} \sup _{s \in[0, T]}\|P F(X(s))\|_{L^{2 p}\left(\Omega ; \dot{H}^{2}\right)} \\
& \leq C k .
\end{aligned}
$$

When estimating $\mathbb{J}_{13}$, we recall the stochastic Fubini theorem (see [15, Theorem 4.18]) and the Burkholder-Davis-Gundy-type inequality to obtain

$$
\begin{aligned}
\mathbb{J}_{13} & =\left\|\sum_{j=1}^{n} \int_{t_{j-1}}^{t_{j}} \int_{t_{j-1}}^{t_{j}} \chi_{\left[s, t_{j}\right)}(\sigma) E\left(t_{n}-s\right) A P F^{\prime}(X(s)) E\left(t_{j}-\sigma\right) \mathrm{d} W(\sigma) \mathrm{d} s\right\|_{L^{p}(\Omega ; \dot{H})} \\
& =\left\|\sum_{j=1}^{n} \int_{t_{j-1}}^{t_{j}} \int_{t_{j-1}}^{t_{j}} \chi_{\left[s, t_{j}\right)}(\sigma) E\left(t_{n}-s\right) A P F^{\prime}(X(s)) E\left(t_{j}-\sigma\right) \mathrm{d} s \mathrm{~d} W(\sigma)\right\|_{L^{p}(\Omega ; \dot{H})} \\
& \leq\left(\sum_{j=1}^{n} \int_{t_{j-1}}^{t_{j}}\left\|\int_{t_{j-1}}^{t_{j}} \chi_{\left[s, t_{j}\right)}(\sigma) E\left(t_{n}-s\right) A P F^{\prime}(X(s)) E\left(t_{j}-\sigma\right) Q^{\frac{1}{2}} \mathrm{~d} s\right\|_{L^{p}\left(\Omega ; \mathcal{L}_{2}\right)}^{2} \mathrm{~d} \sigma\right)^{\frac{1}{2}},
\end{aligned}
$$

where $\chi_{\left[s, t_{j}\right)}(\cdot)$ stands for the indicator function defined by $\chi_{\left[s, t_{j}\right)}(\sigma)=1$ for $\sigma \in\left[s, t_{j}\right)$ and $\chi_{\left[s, t_{j}\right)}(\sigma)=$ 
0 for $\sigma \notin\left[s, t_{j}\right)$. Further, we employ the Hölder inequality, (2.32) and (2.6) with $\varrho=\frac{4-\gamma}{2}$ to deduce,

$$
\begin{aligned}
\mathbb{J}_{13} & \leq C k^{\frac{1}{2}}\left(\sum_{j=1}^{n} \int_{t_{j-1}}^{t_{j}} \int_{t_{j-1}}^{t_{j}} \sum_{l=1}^{\infty}\left\|E\left(t_{n}-s\right) A P F^{\prime}(X(s)) E\left(t_{j}-\sigma\right) Q^{\frac{1}{2}} e_{l}\right\|_{L^{p}(\Omega ; \dot{H})}^{2} \mathrm{~d} s \mathrm{~d} \sigma\right)^{\frac{1}{2}} \\
& \leq C k^{\frac{1}{2}}\left(\sum_{j=1}^{n} \int_{t_{j-1}}^{t_{j}} \sum_{l=1}^{\infty} \int_{t_{j-1}}^{t_{j}}\left(t_{n}-s\right)^{-\frac{1}{2}}\left\|A^{\frac{1}{2}} P F^{\prime}(X(s)) E\left(t_{j}-\sigma\right) Q^{\frac{1}{2}} e_{l}\right\|_{L^{p}(\Omega ; \dot{H})}^{2} \mathrm{~d} s \mathrm{~d} \sigma\right)^{\frac{1}{2}} \\
& \leq C k^{\frac{1}{2}}\left(\sum_{j=1}^{n} \int_{t_{j-1}}^{t_{j}} \sum_{l=1}^{\infty} \int_{t_{j-1}}^{t_{j}}\left(t_{n}-s\right)^{-\frac{1}{2}}\left(1+\sup _{r \in[0, T]}\|X(r)\|_{L^{2 p}\left(\Omega ; \dot{H}^{2}\right)}^{2}\right)\left\|A^{\frac{1}{2}} E\left(t_{j}-\sigma\right) Q^{\frac{1}{2}} e_{l}\right\|_{L_{6}}^{2} \mathrm{~d} s \mathrm{~d} \sigma\right)^{\frac{1}{2}} \\
& \leq C k^{\frac{1}{2}}\left(\sum_{j=1}^{n} \int_{t_{j-1}}^{t_{j}}\left(t_{n}-s\right)^{-\frac{1}{2}} \mathrm{~d} s \int_{t_{j-1}}^{t_{j}}\left\|A E\left(t_{j}-\sigma\right) Q^{\frac{1}{2}}\right\|_{\mathcal{L}_{2}}^{2} \mathrm{~d} \sigma\right)^{\frac{1}{2}} \\
& \leq C k^{\frac{\gamma}{4}}\left(\int_{0}^{t_{n}}\left(t_{n}-s\right)^{-\frac{1}{2}} \mathrm{~d} s\right)^{\frac{1}{2}}\left\|A^{\frac{\gamma-2}{2}} Q^{\frac{1}{2}}\right\|_{\mathcal{L}_{2}} \\
& \leq C k^{\frac{\gamma}{4}},
\end{aligned}
$$

where $\gamma \in[3,4]$ comes from the assumption (2.11) and $\left\{e_{i}\right\}_{i \in \mathbb{N}}$ is any orthogonal basis basis of $\dot{H}$. To bound the term $\mathbb{J}_{14}$, we use (2.18), (2.19) and (2.16) to infer

$$
\begin{aligned}
\mathbb{J}_{14} & \leq C \sum_{j=1}^{n} \int_{t_{j-1}}^{t_{j}}\left(t_{n}-s\right)^{-\frac{2+\delta_{0}}{4}}\left\|R_{F}\left(X(s), X\left(t_{j}\right)\right)\right\|_{L^{p}\left(\Omega ; L_{1}\right)} \mathrm{d} s \\
& \leq C \sum_{j=1}^{n} \int_{t_{j-1}}^{t_{j}}\left(t_{n}-s\right)^{-\frac{2+\delta_{0}}{4}}\|\| X\left(t_{j}\right)-X(s)\|\| f^{\prime \prime}\left((1-\lambda) X(s)+\lambda X\left(t_{j}\right)\right)\left\|_{L_{4}}\right\| X\left(t_{j}\right)-X(s)\left\|_{L_{4}}\right\|_{L^{p}(\Omega ; \mathbb{R})} \mathrm{d} s \\
& \leq C \sum_{j=1}^{n} \int_{t_{j-1}}^{t_{j}}\left(t_{n}-s\right)^{-\frac{2+\delta_{0}}{4}}\left\|X\left(t_{j}\right)-X(s)\right\|_{L^{4 p}(\Omega ; \dot{H})}\left\|X\left(t_{j}\right)-X(s)\right\|_{L^{4 p}\left(\Omega ; \dot{H}^{1}\right)} \mathrm{d} s \sup _{s \in[0, T]}\left\|f^{\prime \prime}(X(s))\right\|_{L^{2 p}\left(\Omega ; L_{4}\right)} \\
& \leq C k \sup _{s \in[0, T]}\left\|f^{\prime \prime}(X(s))\right\|_{L^{2 p}\left(\Omega ; L_{4}\right)} \int_{0}^{t_{n}}\left(t_{n}-s\right)^{-\frac{2+\delta_{0}}{4}} \mathrm{~d} s \\
& \leq C k
\end{aligned}
$$

where for the first step we followed similar arguments as used in (6.14). This together with (6.14), (6.15) and 6.16) leads to, for $\gamma \in[3,4]$,

$$
\mathbb{J}_{1} \leq C k^{\frac{\gamma}{4}} .
$$

Concerning the term $\mathbb{J}_{2}$, we apply (2.27) and (6.7) with $\varrho=\kappa=\min \{\gamma, r\}$ to get

$$
\mathbb{J}_{2} \leq C\left(h^{\kappa}+k^{\frac{\kappa}{4}}\right)\left\|P F\left(X\left(t_{n}\right)\right)\right\|_{L^{p}\left(\Omega ; \dot{H}^{\kappa-2}\right)} \leq C\left(h^{\kappa}+k^{\frac{\kappa}{4}}\right)\left\|P F\left(X\left(t_{n}\right)\right)\right\|_{L^{p}\left(\Omega ; \dot{H}^{2}\right)} \leq C\left(h^{\kappa}+k^{\frac{\kappa}{4}}\right) .
$$

With regard to $\mathbb{J}_{3}$, after employing (6.4) with $\alpha=\kappa$ and (2.33) with $\beta=2$ one can arrive at

$$
\begin{aligned}
\mathbb{J}_{3} & \leq C\left(h^{\kappa}+k^{\frac{\kappa}{4}}\right) \sum_{j=1}^{n} \int_{t_{j-1}}^{t_{j}}\left(t_{n}-s\right)^{-1}\left\|A^{\frac{\kappa-2}{2}} P\left(F\left(X\left(t_{j}\right)\right)-F\left(X\left(t_{n}\right)\right)\right)\right\|_{L^{p}(\Omega ; \dot{H})} \mathrm{d} s \\
& \leq C\left(h^{\kappa}+k^{\frac{\kappa}{4}}\right) \sum_{j=1}^{n-1} \int_{t_{j-1}}^{t_{j}}\left(t_{n}-s\right)^{-1} t_{n-j}^{\frac{1}{4}} \mathrm{~d} s \\
& \leq C\left(h^{\kappa}+k^{\frac{\kappa}{4}}\right), \quad \kappa=\min \{\gamma, r\}
\end{aligned}
$$


where we also used the facts $\kappa-2=\min \{r-2, \gamma-2\} \leq 2$ and $t_{n-j}^{\frac{1}{4}} \leq\left(t_{n}-s\right)^{\frac{1}{4}}$ for $s \leq t_{j}$. Gathering the above three estimates together results in

$$
\mathbb{J} \leq C\left(h^{\kappa}+k^{\frac{\kappa}{4}}\right), \quad \kappa=\min \{\gamma, r\} .
$$

For the last term $\mathbb{K}$, we utilize (6.5) with $\nu=\kappa$ and the Burkholder-Davis-Gundy inequality to obtain

$$
\mathbb{K} \leq\left(\sum_{j=1}^{n} \int_{t_{j-1}}^{t_{j}}\left\|\Psi_{k, h}\left(t_{n}-s\right) Q^{\frac{1}{2}}\right\|_{\mathcal{L}_{2}}^{2} \mathrm{~d} s\right)^{\frac{1}{2}} \leq C\left(h^{\kappa}|\ln h|+k^{\frac{\kappa}{4}}|\ln k|\right)\left\|A^{\frac{\kappa-2}{2}} Q^{\frac{1}{2}}\right\|_{\mathcal{L}_{2}}, \kappa=\min \{\gamma, r\} .
$$

Now, putting the above estimates together results in

$$
\left\|X\left(t_{n}\right)-\widetilde{X}_{h}^{n}\right\|_{L^{p}(\Omega ; \dot{H})} \leq C\left(h^{\kappa}|\ln h|+k^{\frac{\kappa}{4}}|\ln k|\right), \quad \kappa=\min \{\gamma, r\} .
$$

Next we turn our attention to the error $\widetilde{e}_{h}^{n}:=X_{h}^{n}-\widetilde{X}_{h}^{n}$, obeying

$$
\widetilde{e}_{h}^{n}-\widetilde{e}_{h}^{n-1}+k A_{h}^{2} \widetilde{e}_{h}^{n}=-k A_{h} P_{h} F\left(X_{h}^{n}\right)+k A_{h} P_{h} F\left(X\left(t_{n}\right)\right), \quad \widetilde{e}_{h}^{0}=0 .
$$

Equivalently, this can be reformulated as

$$
\widetilde{e}_{h}^{n}=k \sum_{j=1}^{n} A_{h} E_{k, h}^{n-j+1} P_{h}\left(F\left(X\left(t_{j}\right)\right)-F\left(X_{h}^{j}\right)\right) .
$$

Before proceeding further, we need to bound the term $\left\|A_{h} \widetilde{X}_{h}^{n}\right\|_{L^{p}(\Omega ; \dot{H})}$. Owing to (2.27), (5.6), (5.9), (3.10) and the Burkholder-Davis-Gundy inequality, one can derive, for any $n \in\{1,2, \cdots, N\}$,

$$
\begin{aligned}
\left\|A_{h} \widetilde{X}_{h}^{n}\right\|_{L^{p}(\Omega ; \dot{H})} \leq & \left\|A_{h} E_{k, h}^{n} P_{h} X_{0}\right\|_{L^{p}(\Omega ; \dot{H})}+k \sum_{j=1}^{n}\left\|A_{h}^{2} E_{k, h}^{n-j+1} P_{h} P F\left(X\left(t_{j}\right)\right)\right\|_{L^{p}(\Omega ; \dot{H})} \\
& +\left\|\sum_{j=1}^{n} A_{h} E_{k, h}^{n-j+1} P_{h} \Delta W^{j}\right\|_{L^{p}(\Omega ; \dot{H})} \\
\leq & C\left\|A_{h} P_{h} X_{0}\right\|_{L^{p}(\Omega ; \dot{H})}+C k \sum_{j=1}^{n} t_{n-j+1}^{-\frac{1}{2}}\left\|A_{h} P_{h} P F\left(X\left(t_{j}\right)\right)\right\|_{L^{p}(\Omega ; \dot{H})} \\
& +C\left(k \sum_{j=1}^{n}\left\|A_{h} E_{k, h}^{n-j+1} P_{h} Q^{\frac{1}{2}}\right\|_{\mathcal{L}_{2}}^{2}\right)^{\frac{1}{2}} \\
\leq & C\left(1+\left\|X_{0}\right\|_{L^{p}\left(\Omega ; \dot{H}^{2}\right)}+k \sum_{j=1}^{n} t_{n-j+1}^{-\frac{1}{2}} \sup _{s \in[0, T]}\|P F(X(s))\|_{L^{p}\left(\Omega ; \dot{H}^{2}\right)}+\left\|Q^{\frac{1}{2}}\right\|_{\mathcal{L}_{2}}\right)<\infty
\end{aligned}
$$

Multiplying both sides of (6.18) by $A_{h}^{-1} \widetilde{e}_{h}^{n}$ yields

$$
\left(\widetilde{e}_{h}^{n}-\widetilde{e}_{h}^{n-1}, A_{h}^{-1} \widetilde{e}_{h}^{n}\right)+k\left(\nabla \widetilde{e}_{h}^{n}, \nabla \widetilde{e}_{h}^{n}\right)=k\left(-F\left(X_{h}^{n}\right)+F\left(\widetilde{X}_{h}^{n}\right), \widetilde{e}_{h}^{n}\right)+k\left(-F\left(\widetilde{X}_{h}^{n}\right)+F\left(X\left(t_{n}\right)\right), \widetilde{e}_{h}^{n}\right) .
$$

Noting that $\widetilde{e}_{h}^{0}=0$ and $\frac{1}{2}\left(\left|\widetilde{e}_{h}^{n}\right|_{-1, h}^{2}-\left|\widetilde{e}_{h}^{n-1}\right|_{-1, h}^{2}\right) \leq\left(\widetilde{e}_{h}^{n}-\widetilde{e}_{h}^{n-1}, A_{h}^{-1} \widetilde{e}_{h}^{n}\right)$, one can follow a similar way as in (4.20) to arrive at

$$
\frac{1}{2}\left(\left|\widetilde{e}_{h}^{n}\right|_{-1, h}^{2}-\left|\widetilde{e}_{h}^{n-1}\right|_{-1, h}^{2}\right)+k\left|\widetilde{e}_{h}^{n}\right|_{1}^{2} \leq \frac{k}{2}\left|\widetilde{e}_{h}^{n}\right|_{1}^{2}+\frac{9 k}{8}\left|\widetilde{e}_{h}^{n}\right|_{-1, h}^{2}+C k\left\|\widetilde{X}_{h}^{n}-X\left(t_{n}\right)\right\|^{2}\left(1+\left|\widetilde{X}_{h}^{n}\right|_{2, h}^{4}+\left|X\left(t_{n}\right)\right|_{2}^{4}\right) .
$$


Summation on $n$ and applying the Gronwall inequality give

$$
\left|\widetilde{e}_{h}^{n}\right|_{-1, h}^{2}+k \sum_{j=1}^{n}\left|\widetilde{e}_{h}^{j}\right|_{1}^{2} \leq C k \sum_{j=1}^{n}\left\|\widetilde{X}_{h}^{j}-X\left(t_{j}\right)\right\|^{2}\left(1+\left|\widetilde{X}_{h}^{j}\right|_{2, h}^{4}+\left|X\left(t_{j}\right)\right|_{2}^{4}\right),
$$

which together with (2.17), 6.20) and (6.17) leads to

$$
\begin{aligned}
\left\|k \sum_{j=1}^{n}\left|\widetilde{e}_{h}^{j}\right|_{1}^{2}\right\|_{L^{p}(\Omega ; \mathbb{R})} & \leq C k \sum_{j=1}^{n}\|\| \widetilde{X}_{h}^{j}-X\left(t_{j}\right)\left\|^{2}\left(1+\left|\widetilde{X}_{h}^{j}\right|_{2, h}^{4}+\left|X\left(t_{j}\right)\right|_{2}^{4}\right)\right\|_{L^{p}(\Omega ; \mathbb{R})} \\
& \leq C k \sum_{j=1}^{n}\left\|\widetilde{X}_{h}^{j}-X\left(t_{j}\right)\right\|_{L^{4 p}(\Omega ; \dot{H})}^{2}\left(1+\left\|A_{h} \widetilde{X}_{h}^{j}\right\|_{L^{8 p}(\Omega ; \dot{H})}^{4}+\left\|X\left(t_{j}\right)\right\|_{L^{8 p}\left(\Omega ; \dot{H}^{2}\right)}^{4}\right) \\
& \leq C\left(h^{\kappa}|\ln h|+k^{\frac{\kappa}{4}}|\ln k|\right)^{2}, \quad \kappa=\min \{\gamma, r\} .
\end{aligned}
$$

Similarly to the semi-discrete case, we use (6.19) to split the error $\left\|\widetilde{e}_{h}^{n}\right\|_{L^{p}(\Omega ; \dot{H})}$ as follows:

$$
\begin{aligned}
\left\|\widetilde{e}_{h}^{n}\right\|_{L^{p}(\Omega ; \dot{H})} \leq & k \sum_{j=1}^{n}\left\|A_{h} E_{k, h}^{n-j+1} P_{h} P\left(F\left(X\left(t_{j}\right)\right)-F\left(\widetilde{X}_{h}^{j}\right)\right)\right\|_{L^{p}(\Omega ; \dot{H})} \\
& +k\left\|\sum_{j=1}^{n} A_{h} E_{k, h}^{n-j+1} P_{h} P\left(F\left(\widetilde{X}_{h}^{j}\right)-F\left(X_{h}^{j}\right)\right)\right\|_{L^{p}(\Omega ; \dot{H})} \\
= & : \mathbb{A}+\mathbb{B} .
\end{aligned}
$$

Similarly to (4.25), we employ (6.17), (5.6), (6.20) and (2.17) to get

$$
\begin{aligned}
\mathbb{A} & \leq C k \sum_{j=1}^{n} t_{n-j+1}^{-\frac{1}{2}}\left\|F\left(X\left(t_{j}\right)\right)-F\left(\widetilde{X}_{h}^{j}\right)\right\|_{L^{p}(\Omega ; \dot{H})} \\
& \leq C k \sum_{j=1}^{n} t_{n-j+1}^{-\frac{1}{2}}\left\|X\left(t_{j}\right)-\widetilde{X}_{h}^{j}\right\|_{L^{2 p}(\Omega ; \dot{H})}\left(1+\left\|X\left(t_{j}\right)\right\|_{L^{4 p}\left(\Omega ; \dot{H}^{2}\right)}^{2}+\left\|A_{h} \widetilde{X}_{h}^{j}\right\|_{L^{4 p}(\Omega ; \dot{H})}^{2}\right) \\
& \leq C\left(h^{\kappa}|\ln h|+k^{\frac{\kappa}{4}}|\ln k|\right) k \sum_{j=1}^{n} t_{n-j+1}^{-\frac{1}{2}}\left(1+\sup _{s \in[0, T]}\|X(s)\|_{L^{4 p}\left(\Omega ; \dot{H}^{2}\right)}^{2}+\sup _{1 \leq j \leq N}\left\|A_{h} \widetilde{X}_{h}^{j}\right\|_{L^{4 p}(\Omega ; \dot{H})}^{2}\right) \\
& \leq C\left(h^{\kappa}|\ln h|+k^{\frac{\kappa}{4}}|\ln k|\right), \quad \kappa=\min \{\gamma, r\} .
\end{aligned}
$$

For the term $\mathbb{B}$, similar techniques used in (4.26) help us to show

$$
\left\|A_{h}^{\frac{1}{2}} P_{h} P\left(F\left(\widetilde{X}_{h}^{j}\right)-F\left(X_{h}^{j}\right)\right)\right\| \leq C\left|\widetilde{X}_{h}^{j}-X_{h}^{j}\right|_{1}\left(1+\left\|A_{h} \widetilde{X}_{h}^{j}\right\|^{2}+\left\|A_{h} X_{h}^{j}\right\|^{2}\right) .
$$


Combining this with (6.21), (6.20) and (5.2) enables us to derive

$$
\begin{aligned}
\mathbb{B} & \leq\left\|k \sum_{j=1}^{n} t_{n-j+1}^{-\frac{1}{4}}\right\| A_{h}^{\frac{1}{2}} P_{h} P\left(F\left(\widetilde{X}_{h}^{j}\right)-F\left(X_{h}^{j}\right)\right)\|\|_{L^{p}(\Omega ; \mathbb{R})} \\
& \leq C\left\|k \sum_{j=1}^{n} t_{n-j+1}^{-\frac{1}{4}}\left|\widetilde{e}_{h}^{j}\right|_{1}\left(1+\left\|A_{h} \widetilde{X}_{h}^{j}\right\|^{2}+\left\|A_{h} X_{h}^{j}\right\|^{2}\right)\right\|_{L^{p}(\Omega ; \mathbb{R})} \\
& \leq C\left\|\left(k \sum_{j=1}^{n}\left|\widetilde{e}_{h}^{j}\right|_{1}^{2}\right)^{\frac{1}{2}}\left(k \sum_{j=1}^{n} t_{n-j+1}^{-\frac{1}{2}}\left(1+\left\|A_{h} \widetilde{X}_{h}^{j}\right\|^{4}+\left\|A_{h} X_{h}^{j}\right\|^{4}\right)\right)^{\frac{1}{2}}\right\|_{L^{p}(\Omega ; \mathbb{R})} \\
& \leq C\left\|k \sum_{j=1}^{n}\left|\widetilde{e}_{h}^{j}\right|_{1}^{2}\right\|_{L^{p}(\Omega ; \mathbb{R})}^{\frac{1}{2}}\left\|k \sum_{j=1}^{n} t_{n-j+1}^{-\frac{1}{2}}\left(1+\left\|A_{h} \widetilde{X}_{h}^{j}\right\|^{4}+\left\|A_{h} X_{h}^{j}\right\|^{4}\right)\right\|_{L^{p}(\Omega ; \mathbb{R})}^{\frac{1}{2}} \\
& \leq C\left(h^{\kappa}|\ln h|+k^{\frac{\kappa}{4}}|\ln k|\right), \quad \kappa=\min \{\gamma, r\},
\end{aligned}
$$

which together with (6.23), 6.22) and (6.17) gives the desired assertion (6.8).

In the sequel, we focus on the error $\left\|Y\left(t_{n}\right)-Y_{h}^{n}\right\|_{L^{p}(\Omega ; \dot{H})}$. Similarly to the semi-discrete case, we first consider the error $\left\|Y\left(t_{n}\right)-\widetilde{Y}_{h}^{n}\right\|_{L^{p}(\Omega ; \dot{H})}$, where $\widetilde{Y}_{h}^{n}=A_{h} \widetilde{X}_{h}^{n}+P_{h} P F\left(X\left(t_{n}\right)\right)$. By (6.11) and (1.4),

$$
\begin{aligned}
\left\|Y\left(t_{n}\right)-\widetilde{Y}_{h}^{n}\right\|_{L^{p}(\Omega ; \dot{H})} \leq & \underbrace{\left\|\left(A E\left(t_{n}\right)-A_{h} E_{k, h}^{n} P_{h}\right) X_{0}\right\|_{L^{p}(\Omega ; \dot{H})}+\left\|\left(I-P_{h}\right) P F\left(X\left(t_{n}\right)\right)\right\|_{L^{p}(\Omega ; \dot{H})}}_{\mathbb{L}_{1}} \\
& +\underbrace{\left\|\sum_{j=1}^{n} \int_{t_{j-1}}^{t_{j}} A^{2} E\left(t_{n}-s\right) P F(X(s))-A_{h}^{2} E_{k, h}^{n-j+1} P_{h} P F\left(X\left(t_{j}\right)\right) \mathrm{d} s\right\|_{L^{p}(\Omega ; \dot{H})}}_{\mathbb{L}_{2}} \\
& +\underbrace{\left(\sum_{j=1}^{n} \int_{t_{j-1}}^{t_{j}}\left\|\left(A E\left(t_{n}-s\right)-A_{h} E_{k, h}^{n-j+1} P_{h}\right) Q^{\frac{1}{2}}\right\|_{\mathcal{L}_{2}}^{2} \mathrm{~d} s\right)^{\frac{1}{2}}}_{\mathbb{L}_{3}} .
\end{aligned}
$$

In the same spirit as in (4.29) but employing (6.4) with $\alpha=2$ instead we obtain

$$
\begin{aligned}
\mathbb{L}_{1} & \leq C h^{2} \sup _{s \in[0, T]}\|P F(X(s))\|_{L^{p}\left(\Omega ; \dot{H}^{2}\right)}+C\left(h^{2}+k^{\frac{1}{2}}\right) t_{n}^{-1}\left\|X_{0}\right\|_{L^{p}(\Omega ; \dot{H})} \\
& \leq C\left(h^{2}+k^{\frac{1}{2}}\right)\left(1+t_{n}^{-1}\right) .
\end{aligned}
$$

In order to properly handle $\mathbb{L}_{2}$, we need its further decomposition as follows,

$$
\begin{aligned}
\mathbb{L}_{2} \leq & \left\|\sum_{j=1}^{n} \int_{t_{j-1}}^{t_{j}} A^{2} E\left(t_{n}-s\right) P\left(F(X(s))-F\left(X\left(t_{j}\right)\right)\right) \mathrm{d} s\right\|_{L^{p}(\Omega ; \dot{H})} \\
& +\sum_{j=1}^{n} \int_{t_{j-1}}^{t_{j}} \|\left(A^{2} E\left(t_{n}-s\right)-A_{h}^{2} E_{k, h}^{n-j+1} P_{h}\right) P\left(F\left(X\left(t_{j}\right)-F\left(X\left(t_{n}\right)\right)\right) \|_{L^{p}(\Omega ; \dot{H})} \mathrm{d} s\right. \\
& +\left\|\sum_{j=1}^{n} \int_{t_{j-1}}^{t_{j}}\left(A^{2} E\left(t_{n}-s\right)-A_{h}^{2} E_{k, h}^{n-j+1} P_{h}\right) P F\left(X\left(t_{n}\right)\right) \mathrm{d} s\right\|_{L^{p}(\Omega ; \dot{H})} \\
=: & \mathbb{L}_{21}+\mathbb{L}_{22}+\mathbb{L}_{23} .
\end{aligned}
$$


Thanks to (2.33) with $\beta=1$ and (2.4) with $\mu=\frac{3}{2}$, one can show

$$
\begin{aligned}
\mathbb{L}_{21} & \leq C \sum_{j=1}^{n} \int_{t_{j-1}}^{t_{j}}\left(t_{n}-s\right)^{-\frac{3}{4}}\left\|A^{\frac{1}{2}} P\left(F(X(s))-F\left(X\left(t_{j}\right)\right)\right)\right\|_{L^{p}(\Omega ; \dot{H})} \mathrm{d} s \\
& \leq C k^{\frac{1}{2}} \int_{0}^{t_{n}}\left(t_{n}-s\right)^{-\frac{3}{4}} \mathrm{~d} s \leq C k^{\frac{1}{2}} .
\end{aligned}
$$

Similarly to (4.30), using (2.33) with $\beta=2$, (6.4) with $\alpha=2$, (5.6) with $\mu=2$ and (3.6) implies

$$
\begin{aligned}
\mathbb{L}_{22} \leq & \sum_{j=1}^{n-1} \int_{t_{j-1}}^{t_{j}}\left\|\Phi_{k, h}\left(t_{n}-s\right) A P\left(F\left(X\left(t_{j}\right)\right)-F\left(X\left(t_{n}\right)\right)\right)\right\|_{L^{p}(\Omega ; \dot{H})} \mathrm{d} s \\
& +\sum_{j=1}^{n-1} \int_{t_{j-1}}^{t_{j}}\left\|A_{h}^{2} E_{k, h}^{n-j+1} P_{h}\left(I-R_{h}\right) P\left(F\left(X\left(t_{j}\right)\right)-F\left(X\left(t_{n}\right)\right)\right)\right\|_{L^{p}(\Omega ; \dot{H})} \mathrm{d} s \\
\leq & C\left(h^{2}+k^{\frac{1}{2}}\right) \sum_{j=1}^{n-1} \int_{t_{j-1}}^{t_{j}}\left(\left(t_{n}-s\right)^{-1}+t_{n-j+1}^{-1}\right)\left\|A P\left(F\left(X\left(t_{j}\right)\right)-F\left(X\left(t_{n}\right)\right)\right)\right\|_{L^{p}(\Omega ; \dot{H})} \mathrm{d} s \\
\leq & C\left(h^{2}+k^{\frac{1}{2}}\right) \sum_{j=1}^{n-1} k\left(t_{n-j}^{-1+\frac{1}{4}}+t_{n-j+1}^{-1+\frac{1}{4}}\right) \\
\leq & C\left(h^{2}+k^{\frac{1}{2}}\right) .
\end{aligned}
$$

Similarly as before, we utilize (3.5), (5.8), (2.27), (6.7) with $\varrho=2$ and (3.6) to bound $\mathbb{L}_{23}$ as follows,

$$
\begin{aligned}
\mathbb{L}_{23} & \leq\left\|\int_{0}^{t_{n}} \Phi_{k, h}\left(t_{n}-s\right) A P F\left(X\left(t_{n}\right)\right) \mathrm{d} s\right\|_{L^{p}(\Omega ; \dot{H})}+\left\|\sum_{j=1}^{n} k A_{h}^{2} E_{k, h}^{n-j+1} P_{h}\left(R_{h}-I\right) P F\left(X\left(t_{n}\right)\right)\right\|_{L^{p}(\Omega ; \dot{H})} \\
& \leq C\left(h^{2}+k^{\frac{1}{2}}\right)\left\|P F\left(X\left(t_{n}\right)\right)\right\|_{L^{p}\left(\Omega ; \dot{H}^{2}\right)}+C\left\|\left(R_{h}-I\right) P F\left(X\left(t_{n}\right)\right)\right\|_{L^{p}(\Omega ; \dot{H})} \\
& \leq C\left(h^{2}+k^{\frac{1}{2}}\right) \sup _{s \in[0, T]}\|P F(X(s))\|_{L^{p}\left(\Omega ; \dot{H}^{2}\right)} .
\end{aligned}
$$

Putting the above three estimates together ensures

$$
\mathbb{L}_{2} \leq C\left(h^{2}+k^{\frac{1}{2}}\right) .
$$

At the moment we start to estimate the term $\mathbb{L}_{3}$. In the light of (6.6) with $\mu=\gamma-2$, we derive

$$
\mathbb{L}_{3} \leq\left(\sum_{j=1}^{n} \int_{t_{j-1}}^{t_{j}}\left\|\Phi_{k, h}\left(t_{n}-s\right) Q^{\frac{1}{2}}\right\|_{\mathcal{L}_{2}}^{2} \mathrm{~d} s\right)^{\frac{1}{2}} \leq C\left(h^{\gamma-2}|\ln h|+k^{\frac{\gamma-2}{4}}|\ln k|\right)\left\|A^{\frac{\gamma-2}{2}} Q^{\frac{1}{2}}\right\|_{\mathcal{L}_{2}},
$$

which together with (6.28) and (6.26) shows

$$
\left\|Y\left(t_{n}\right)-\widetilde{Y}_{h}^{n}\right\|_{L^{p}(\Omega ; \dot{H})} \leq C\left(h^{\gamma-2}|\ln h|+k^{\frac{\gamma-2}{4}}|\ln k|\right)\left(1+t_{n}^{-1}\right) .
$$

Now it remains to bound $\left\|\widetilde{Y}_{h}^{n}-Y_{h}^{n}\right\|_{L^{p}(\Omega ; \dot{H})}$. Using the same arguments as in (4.36) promises

$$
\begin{aligned}
& \left\|P\left(F\left(X\left(t_{j}\right)\right)-F\left(X_{h}^{j}\right)\right)\right\|_{L^{p}(\Omega ; \dot{H})} \\
& \quad \leq C\left(1+\sup _{s \in[0, T]}\|X(s)\|_{L^{4 p}\left(\Omega ; \dot{H}^{2}\right)}^{2}+\sup _{1 \leq j \leq N}\left\|A_{h} X_{h}^{j}\right\|_{L^{4 p}(\Omega ; H)}^{2}\right)\left\|X\left(t_{j}\right)-X_{h}^{j}\right\|_{L^{2 p}(\Omega ; \dot{H})} \\
& \quad \leq C\left(h^{\kappa}|\ln h|+k^{\frac{\kappa}{4}}|\ln k|\right), \quad \kappa=\min \{\gamma, r\} .
\end{aligned}
$$


Combining this with (5.6) with $\mu=\frac{1}{2}$, the inverse inequality (3.9) and the fact $t_{n-j+1}^{-1} \leq C k^{-1}$ helps us to arrive at

$$
\begin{aligned}
\left\|\widetilde{Y}_{h}^{n}-Y_{h}^{n}\right\|_{L^{p}(\Omega ; \dot{H})} \leq & \sum_{j=1}^{n} k\left\|E_{k, h}^{n-j+1} A_{h}^{2} P_{h} P\left(F\left(X\left(t_{j}\right)\right)-F\left(X_{h}^{j}\right)\right)\right\|_{L^{p}(\Omega ; \dot{H})} \\
& +\left\|P\left(F\left(X\left(t_{n}\right)\right)-F\left(X_{h}^{n}\right)\right)\right\|_{L^{p}(\Omega ; \dot{H})} \\
\leq & C \min \left\{h^{-1}, k^{-\frac{1}{4}}\right\} \sum_{j=1}^{n} k t_{n-j+1}^{-\frac{3}{4}}\left\|P\left(F\left(X\left(t_{j}\right)\right)-F\left(X_{h}^{j}\right)\right)\right\|_{L^{p}(\Omega ; \dot{H})} \\
& +C\left(h^{\kappa}|\ln h|+k^{\frac{\kappa}{4}}|\ln k|\right) \\
\leq & C\left(h^{\kappa-1}|\ln h|+k^{\frac{\kappa-1}{4}}|\ln k|\right), \quad \kappa=\min \{\gamma, r\} .
\end{aligned}
$$

Putting (6.29) and (6.30) together finally gives (6.9), as required.

7. Numerical experiments. Some numerical tests are presented in this section to illustrate the previous findings. We consider the following Cahn-Hilliard-Cook equation in one dimension,

$$
\begin{cases}\frac{\partial u}{\partial t}=\frac{\partial^{2} w}{\partial x^{2}}+\dot{W}, & t \in(0, T], x \in(0,1), \\ w=-\frac{\partial^{2} u}{\partial x^{2}}+u-u^{3}, & x \in(0,1), \\ u(0, x)=\cos (\pi x), & x \in(0,1), \\ \left.\frac{\partial u}{\partial x}\right|_{x=0}=\left.\frac{\partial u}{\partial x}\right|_{x=1}=0, & t \in(0, T], \\ \left.\frac{\partial w}{\partial x}\right|_{x=0}=\left.\frac{\partial w}{\partial x}\right|_{x=1}=0, & t \in(0, T],\end{cases}
$$

where $\{W(t)\}_{t \in[0, T]}$ is a standard $Q$-Wiener process in $\dot{H}$, with a covariance operator $Q=A^{-1.5005}$. Here $-A$ is the Laplacian with homogeneous Neumann boundary conditions and the condition (2.11) is fulfilled with $\gamma=3$. We aim to perform mean-square approximations of the exact solution $(u, w)$ to (7.1) at the endpoint $T=1$. To do so we take a piecewise linear finite element method for the spatial discretization and a backward Euler method for the temporal discretization. The expectations are approximated by computing averages over $M=100$ samples. The exact solutions $(u, w)$, not available at hand, are computed by numerical ones with small step-sizes $h_{\text {exact }}$ and $k_{\text {exact }}$.

At first, we test the convergence rates for the spatial discretizations. The "true" solutions $(u, w)$ are computed using $h_{\text {exact }}=2^{-5}$ and $k_{\text {exact }}=2^{-20}$. We carry out numerical simulations with three different spatial step-sizes $h=2^{-i}, i=1,2,3$ and present the resulting mean-square errors (solid lines) in Figure 7.1. As expected, convergence rates of order 2 and order 1 are detected for the concentration and the chemical potential, respectively. This is consistent with the previous theoretical findings in Theorem 4.2 when $\gamma=3$ and $r=2$. Next we turn to the temporal discretizaton and fix $h=2^{-7}$ for the FEM semi-discretization, whose "true" solutions $\left(u_{h}, w_{h}\right)$ are computed using $k_{\text {exact }}=2^{-14}$. Similarly, we present in Figure 7.2 errors due to the temporal discretizatons using five time stepsizes $k=2^{-i}, i=6,7,8,9,10$. Comparing the slopes of the error (solid) lines with those of the reference (dashed) lines, one can see, temporal approximation errors decrease at a slope close to order $\frac{1}{2}$ for the concentration and order $\frac{1}{4}$ for the chemical potential, which agree with the theoretical results.

8. Appendix: Proof of Lemma 6.1, The estimates (6.3) and (6.5) can be proved by a simple modification of the proof of [30, Theorem 2.2]. In order to validate (6.4), one can first use (2.4) with $\mu=2$, (2.5) with $\nu=\frac{\alpha}{2}$ and (4.4) to get, for $t \in\left[t_{n-1}, t_{n}\right)$,

$$
\begin{aligned}
\left\|\Phi_{k, h}(t) v\right\| & \leq\left\|A\left(E(t)-E\left(t_{n}\right)\right) v\right\|+\left\|\left(A E\left(t_{n}\right)-A_{h} E_{h}\left(t_{n}\right) P_{h}\right) v\right\|+\left\|A_{h}\left(E_{h}\left(t_{n}\right)-E_{k, h}^{n}\right) P_{h} v\right\| \\
& \leq\left\|A^{2} E(t) A^{-\frac{\alpha}{2}}\left(I-E\left(t_{n}-t\right)\right) A^{\frac{\alpha-2}{2}} v\right\|+C t_{n}^{-1} h^{\alpha}|v|_{\alpha-2}+\left\|A_{h}\left(E_{h}\left(t_{n}\right)-E_{k, h}^{n}\right) P_{h} v\right\| \\
& \leq C t^{-1}\left(h^{\alpha}+k^{\frac{\alpha}{4}}\right)|v|_{\alpha-2}+\left\|A_{h}\left(E_{h}\left(t_{n}\right)-E_{k, h}^{n}\right) P_{h} v\right\|,
\end{aligned}
$$



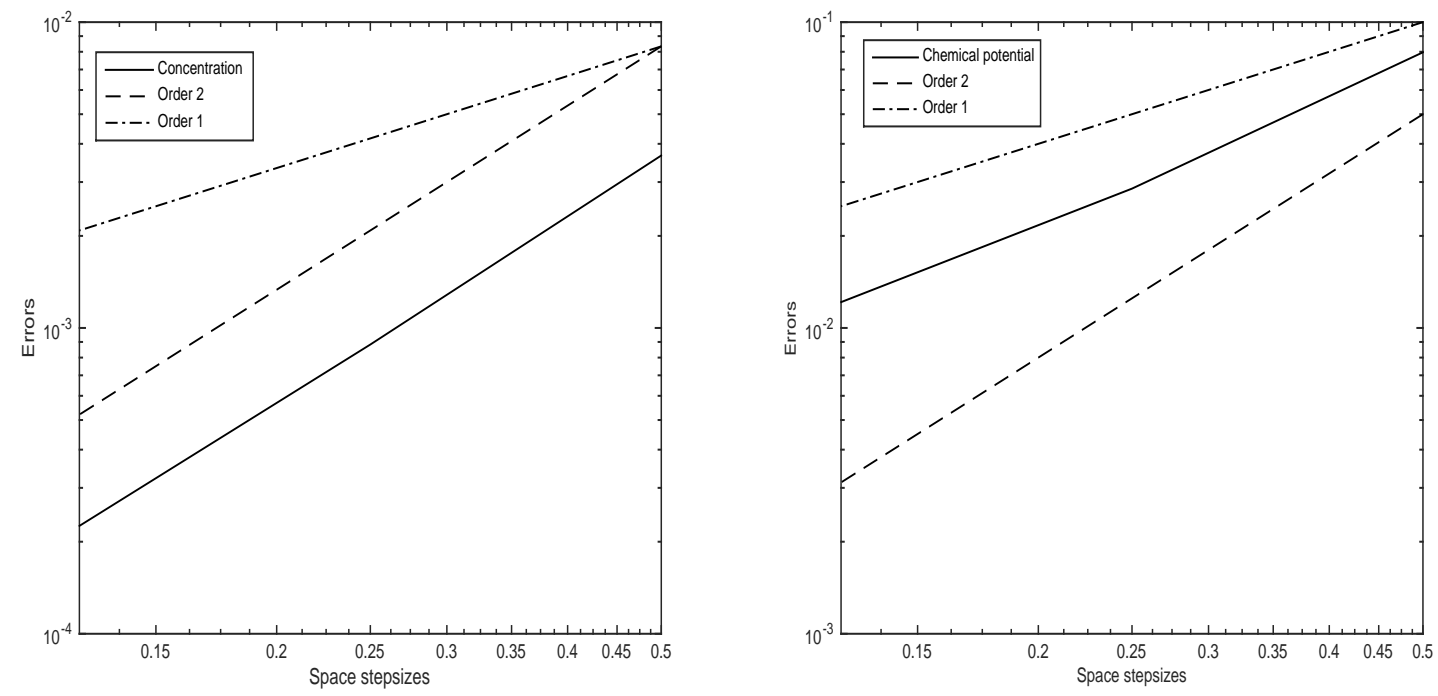

FIG. 7.1. Mean-square convergence rates for the spatial discretizations
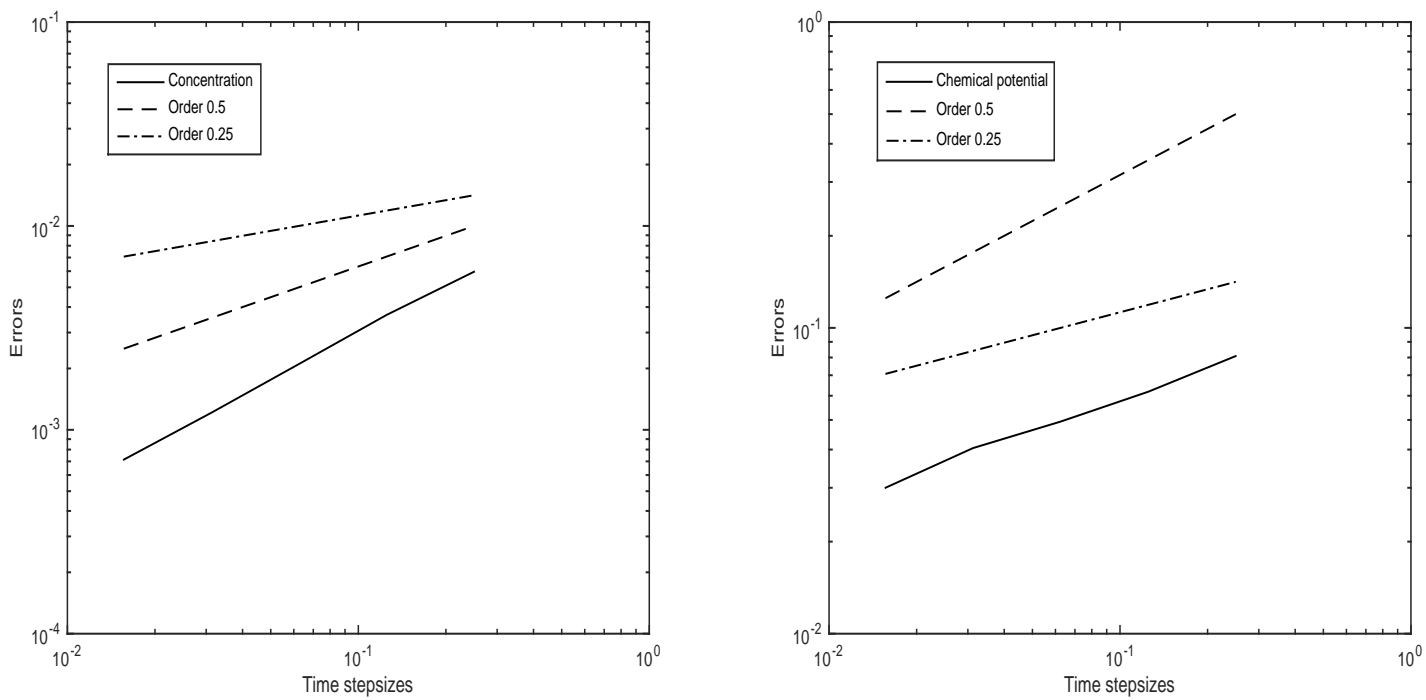

FIG. 7.2. Mean-square convergence rates for the temporal discretizations

where

$$
\left\|A_{h}\left(E_{h}\left(t_{n}\right)-E_{k, h}^{n}\right) P_{h} v\right\| \leq C t_{n}^{-1}\left\|A_{h}^{-1} P_{h} v\right\|,
$$

due to the use of (3.16) with $\mu=2$ and (5.6) with $\mu=2$. On the other hand, 31, Theorem 4.4] shows

$$
\left\|A_{h}\left(E_{h}\left(t_{n}\right)-E_{k, h}^{n}\right) P_{h} v\right\| \leq C k t_{n}^{-1}\left\|A_{h} P_{h} v\right\| .
$$


An interpolation between these two estimates shows, for $\beta \in[0,4]$ and $t \in\left[t_{n-1}, t_{n}\right)$,

$$
\left\|A_{h}\left(E_{h}\left(t_{n}\right)-E_{k, h}^{n}\right) P_{h} v\right\| \leq C t_{n}^{-1} k^{\frac{\beta}{4}}\left\|A_{h}^{\frac{\beta-2}{2}} P_{h} v\right\| \leq C t^{-1} k^{\frac{\beta}{4}}\left\|A_{h}^{\frac{\beta-2}{2}} P_{h} v\right\|,
$$

which, after assigning $\beta=\alpha \in[1, r]$ and considering (8.1) and (3.13), implies (6.4). Repeating the same arguments in the proof of (4.6), we can show (6.6). Next we prove (6.7). Note first that, for $t \in\left[t_{n}, t_{n+1}\right), n \geq 0$,

$$
\left\|\int_{0}^{t} \Phi_{k, h}(s) v \mathrm{~d} s\right\| \leq\left\|\int_{t_{n}}^{t} \Phi_{k, h}(s) v \mathrm{~d} s\right\|+\left\|\int_{0}^{t_{n}} \Phi_{k, h}(s) v \mathrm{~d} s\right\| .
$$

By virtue of (3.11), (2.5) with $\nu=\frac{\varrho}{2}$ and (5.6) with $\mu=\frac{4-\varrho}{2}$, we acquire

$$
\begin{aligned}
\left\|\int_{t_{n}}^{t} \Phi_{k, h}(s) v \mathrm{~d} s\right\| & =\left\|A^{-1}\left(E(t)-E\left(t_{n}\right)\right) v\right\|+\left(t-t_{n}\right)\left\|A_{h} E_{k, h}^{n+1} P_{h} v\right\| \\
& \leq C\left\|A^{-\frac{\varrho}{2}}\left(I-E\left(t-t_{n}\right)\right) A^{\frac{\varrho-2}{2}} v\right\|+\left(t-t_{n}\right)\left\|A_{h}^{\frac{4-\varrho}{2}} E_{k, h}^{n+1} A_{h}^{\frac{\varrho-2}{2}} P_{h} v\right\| \\
& \leq C\left(k^{\frac{\varrho}{4}}+\left(t-t_{n}\right) t_{n+1}^{-\frac{4-\varrho}{4}}\right)|v|_{\varrho-2} \\
& \leq C k^{\frac{\varrho}{4}}|v|_{\varrho-2} .
\end{aligned}
$$

Further, owing to (4.7) we obtain

$$
\begin{aligned}
\left\|\int_{0}^{t_{n}} \Phi_{k, h}(s) v \mathrm{~d} s\right\| \leq & \left\|\int_{0}^{t_{n}} \Phi_{h}(s) v \mathrm{~d} s\right\|+\left\|\sum_{j=1}^{n} \int_{t_{j-1}}^{t_{j}}\left(E_{h}(s)-E_{h}\left(t_{j}\right)\right) A_{h} P_{h} v \mathrm{~d} s\right\| \\
& +\left\|\sum_{j=1}^{n} \int_{t_{j-1}}^{t_{j}}\left(E_{h}\left(t_{j}\right)-E_{k, h}^{j}\right) A_{h} P_{h} v \mathrm{~d} s\right\| \\
\leq & C h^{\varrho}|v|_{\varrho-2}+\left\|\sum_{j=1}^{n} \int_{t_{j-1}}^{t_{j}}\left(E_{h}(s)-E_{h}\left(t_{j}\right)\right) A_{h} P_{h} v \mathrm{~d} s\right\| \\
& +\left\|k \sum_{j=1}^{n}\left(E_{h}\left(t_{j}\right)-E_{k, h}^{j}\right) A_{h} P_{h} v\right\|,
\end{aligned}
$$

where using Parseval's identity and the fact $\lambda_{i, h}^{-\frac{\varrho}{2}}\left(1-e^{-\left(t_{j}-s\right) \lambda_{i, h}^{2}}\right) \leq C k^{\frac{\varrho}{4}}, 0 \leq \varrho \leq 4$ gives

$$
\begin{aligned}
\| \sum_{j=1}^{n} \int_{t_{j-1}}^{t_{j}}\left(E_{h}(s)\right. & \left.-E_{h}\left(t_{j}\right)\right) A_{h} P_{h} v \mathrm{~d} s\left\|^{2}=\right\| \sum_{j=1}^{n} \sum_{i=1}^{\mathcal{N}_{h}} \int_{t_{j-1}}^{t_{j}}\left(e^{-s \lambda_{i, h}^{2}}-e^{-t_{j} \lambda_{i, h}^{2}}\right) \lambda_{i, h}\left(P_{h} v, e_{i, h}\right) e_{i, h} \mathrm{~d} s \|^{2} \\
& =\sum_{i=1}^{\mathcal{N}_{h}}\left[\left|\sum_{j=1}^{n} \int_{t_{j-1}}^{t_{j}} \lambda_{i, h}^{2} e^{-s \lambda_{i, h}^{2}} \lambda_{i, h}^{-\frac{\varrho}{2}}\left(1-e^{-\left(t_{j}-s\right) \lambda_{i, h}^{2}}\right) \mathrm{d} s\right|^{2} \lambda_{i, h}^{\varrho-2}\left(P_{h} v, e_{i, h}\right)^{2}\right] \\
& \leq C k^{\frac{\varrho}{2}} \sum_{i=1}^{\mathcal{N}_{h}}\left|\int_{0}^{t_{n}} \lambda_{i, h}^{2} e^{-s \lambda_{i, h}^{2}} \mathrm{~d} s\right|^{2} \lambda_{i, h}^{\varrho-2}\left(P_{h} v, e_{i, h}\right)^{2} \\
& \leq C k^{\frac{\varrho}{2}} \sum_{i=1}^{\mathcal{N}_{h}} \lambda_{i, h}^{\varrho-2}\left(P_{h} v, e_{i, h}\right)^{2} \\
& =C k^{\frac{\varrho}{2}}\left\|A_{h}^{\frac{\varrho-2}{2}} P_{h} v\right\|^{2} \\
& \leq C k^{\frac{\varrho}{2}}|v|_{\varrho-2}^{2} .
\end{aligned}
$$


Likewise,

$$
\begin{aligned}
\left\|k \sum_{j=1}^{n}\left(E_{h}\left(t_{j}\right)-E_{k, h}^{j}\right) A_{h} P_{h} v\right\|^{2} & =\left\|\sum_{j=1}^{n} k \sum_{i=1}^{\mathcal{N}_{h}}\left(e^{-t_{j} \lambda_{i, h}^{2}}-r\left(k \lambda_{i, h}^{2}\right)^{j}\right) \lambda_{i, h}\left(P_{h} v, e_{i, h}\right) e_{i, h}\right\|^{2} \\
& =\sum_{i=1}^{\mathcal{N}_{h}}\left|\sum_{j=1}^{n} k\left(e^{-t_{j} \lambda_{i, h}^{2}}-r\left(k \lambda_{i, h}^{2}\right)^{j}\right) \lambda_{i, h}\right|^{2}\left(P_{h} v, e_{i, h}\right)^{2} .
\end{aligned}
$$

Here we consider two possibilities: either $k \lambda_{i, h}^{2} \leq 1$ or $k \lambda_{i, h}^{2}>1$. For all summands with $k \lambda_{i, h}^{2} \leq 1$, we rely on (5.5) to get

$$
\begin{aligned}
\left|k \sum_{j=1}^{n}\left(e^{-j k \lambda_{i, h}^{2}}-r\left(k \lambda_{i, h}^{2}\right)^{j}\right) \lambda_{i, h}\right| & \leq C \lambda_{i, h}^{5} k^{2} \sum_{j=1}^{n} j k e^{-c(j-1) k \lambda_{i, h}^{2}} \leq C \lambda_{i, h}^{5} k \int_{0}^{\infty}(r+k) e^{-c r \lambda_{i, h}^{2}} \mathrm{~d} r \\
& \leq C \lambda_{i, h}^{5} k\left(\frac{1}{\left(c \lambda_{i, h}^{2}\right)^{2}}+\frac{k}{c \lambda_{i, h}^{2}}\right) \leq C \lambda_{i, h}^{\frac{\rho-2}{2}} k^{\frac{o}{4}} .
\end{aligned}
$$

For all summands with $k \lambda_{i, h}^{2}>1$, utilizing the fact $\sup _{s \in[0, \infty)} s e^{-s}<\infty$ yields

$$
\begin{aligned}
\left|k \sum_{j=1}^{n}\left(e^{-j k \lambda_{i, h}^{2}}-r\left(k \lambda_{i, h}^{2}\right)^{j}\right) \lambda_{i, h}\right| & \leq C\left(k \lambda_{i, h} e^{-k \lambda_{i, h}^{2}} \sum_{j=1}^{n} e^{-(j-1)}+\frac{k \lambda_{i, h}}{1+k \lambda_{i, h}^{2}} \sum_{j=1}^{n} 2^{-j+1}\right) \\
& \leq C k^{\frac{\rho}{4}} \lambda_{i, h}^{\frac{\rho-2}{2}}\left(k \lambda_{i, h}^{2}\right)^{1-\frac{\rho}{4}}\left(e^{-k \lambda_{i, h}^{2}}+\frac{1}{k \lambda_{i, h}^{2}}\right) \\
& \leq C k^{\frac{o}{4}} \lambda_{i, h}^{\frac{\rho-2}{2}} .
\end{aligned}
$$

This together with (3.10) and (3.13) proves

$$
\left\|k \sum_{j=1}^{n}\left(E_{h}\left(t_{j}\right)-E_{k, h}^{j}\right) A_{h} P_{h} v\right\|^{2} \leq C k^{\frac{\varrho}{2}} \sum_{i=1}^{\mathcal{N}_{h}} \lambda_{i, h}^{\varrho-2}\left(P_{h} v, e_{i, h}\right)^{2} \leq C k^{\frac{\varrho}{2}}\left\|A_{h}^{\frac{\varrho-2}{2}} P_{h} v\right\|^{2} \leq C k^{\frac{\varrho}{2}}|v|_{\varrho-2}^{2} .
$$

Finally, plugging (8.4)-(8.6) and (8.7) into (8.3) shows (6.7) and thus finishes the proof.

\section{REFERENCES}

[1] Sebastian Becker, Benjamin Gess, Arnulf Jentzen, and Peter E Kloeden, Strong convergence rates for explicit space-time discrete numerical approximations of stochastic Allen-Cahn equations, arXiv preprint arXiv:1711.02423, (2017).

[2] Sebastian Becker And Arnulf Jentzen, Strong convergence rates for nonlinearity-truncated Euler-type approximations of stochastic Ginzburg-Landau equations, Stochastic Processes and their Applications, 129 (2019), pp. $28-69$.

[3] Charles-Edouard Bréhier, Jianbo Cui, and Jialin Hong, Strong convergence rates of semi-discrete splitting approximations for stochastic Allen-Cahn equation, IMA J. Numer. Anal., 39 (2019), pp. 2096-2134.

[4] Charles-Edouard Bréhier and Ludovic Goudenège, Analysis of some splitting schemes for the stochastic Allen-Cahn equation, Discrete Contin. Dyn. Syst. B, 24 (2019), pp. 4169-4190.

[5] Charles-Edouard Bréhier and Ludovic Goudenège, Weak convergence rates of splitting schemes for the stochastic Allen-Cahn equation, BIT Numerical Mathematics, (2019).

[6] John W CAhn, On spinodal decomposition, Acta metallurgica, 9 (1961), pp. 795-801.

[7] John W CAhn And John E Hilliard, Free energy of a nonuniform system. i. interfacial free energy, The Journal of chemical physics, 28 (1958), pp. 258-267.

[8] - Spinodal decomposition: A reprise, Acta Metallurgica, 19 (1971), pp. 151-161.

[9] Stuart Campbell and Gabriel Lord, Adaptive time-stepping for stochastic partial differential equations with non-Lipschitz drift, arXiv preprint arXiv:1812.09036, (2018). 
[10] Caroline Cardon-Weber, Implicit approximation scheme for the cahn-hilliard stochastic equation, Preprint, Laboratoire des Probabilités et Modelelés Aléatoires, Université Paris V, (2000).

[11] Jianbo Cui And Jialin Hong, Strong and weak convergence rates of a spatial approximation for stochastic partial differential equation with one-sided lipschitz coefficient, SIAM Journal on Numerical Analysis, 57 (2019), pp. $1815-1841$.

[12] Jianbo Cui, Jialin Hong, and Liying Sun, Strong convergence rate of a full discretization for stochastic CahnHilliard equation driven by space-time white noise, arXiv preprint arXiv:1812.06289, (2018).

[13] —- Weak convergence and invariant measure of a full discretization for non-globally Lipschitz parabolic SPDE, arXiv preprint arXiv:1811.04075, (2018).

[14] Giuseppe Da Prato and Arnaud Debussche, Stochastic cahn-hilliard equation, Nonlinear Analysis: Theory, Methods \& Applications, 26 (1996), pp. 241-263.

[15] Giuseppe Da Prato and Jerzy Zabczyk, Stochastic equations in infinite dimensions, Cambridge university press, 2014.

[16] Charles M. Elliott and Stig Larsson, Error estimates with smooth and nonsmooth data for a finite element method for the Cahn-Hilliard equation, Mathematics of Computation, 58 (1992), pp. 603-630.

[17] Xiaobing Feng, Yukun Li, And Yi Zhang, Finite element methods for the stochastic Allen-Cahn equation with gradient-type multiplicative noise, SIAM Journal on Numerical Analysis, 55 (2017), pp. 194-216.

[18] - Strong convergence of a fully discrete finite element method for a class of semilinear stochastic partial differential equations with multiplicative noise, arXiv preprint arXiv:1811.05028, (2018).

[19] Daisuke Furihata, Mihály Kovács, Stig Larsson, and Fredrik Lindgren, Strong convergence of a fully discrete finite element approximation of the stochastic cahn-hilliard equation, SIAM Journal on Numerical Analysis, 56 (2018), pp. 708-731.

[20] István Gyöngy, Sotirios Sabanis, And David ŠišKa, Convergence of tamed euler schemes for a class of stochastic evolution equations, Stochastics and Partial Differential Equations: Analysis and Computations, 4 (2016), pp. 225-245.

[21] Martin Hutzenthaler And ARnulf Jentzen, On a perturbation theory and on strong convergence rates for stochastic ordinary and partial differential equations with non-globally monotone coefficients, arXiv preprint arXiv:1401.0295, to appear in The Annals of Probability, (2020).

[22] Arnulf Jentzen and Primož Pušnik, Exponential moments for numerical approximations of stochastic partial differential equations, Stochastics and Partial Differential Equations: Analysis and Computations, 6 (2018), pp. 565-617.

[23] - Strong convergence rates for an explicit numerical approximation method for stochastic evolution equations with non-globally lipschitz continuous nonlinearities, arXiv preprint arXiv:1504.03523, to appear in IMA Journal of Numerical Analysis, (2020).

[24] Jentzen A., Kloeden P.E., Taylor Approximations for Stochastic Partial Differential Equations, SIAM, Philadelphia, 2011.

[25] Mihály Kovács, Stig Larsson, and Fredrik Lindgren, On the backward Euler approximation of the stochastic Allen-Cahn equation, Journal of Applied Probability, 52 (2015), pp. 323-338.

[26] - On the discretisation in time of the stochastic Allen-Cahn equation, Mathematische Nachrichten, 291 (2018), pp. 966-995.

[27] Mihály Kovács, Stig Larsson, and Ali Mesforush, Finite element approximation of the cahn-hilliard-cook equation, SIAM Journal on Numerical Analysis, 49 (2011), pp. 2407-2429.

[28] Mihaly Kovacs, Stig Larsson, and Ali Mesforush, Erratum: Finite element approximation of the cahnhilliard-cook equation, SIAM Journal on Numerical Analysis, 52 (2014), pp. 2594-2597.

[29] Raphael Kruse, Strong and weak approximation of semilinear stochastic evolution equations, Springer, 2014.

[30] Stig Larsson and Ali Mesforush, Finite-element approximation of the linearized cahn-hilliard-cook equation, IMA journal of numerical analysis, 31 (2011), pp. 1315-1333.

[31] Stig Larsson, Vidar Thomée, and Lars B Wahlbin, Finite-element methods for a strongly damped wave equation, IMA journal of numerical analysis, 11 (1991), pp. 115-142.

[32] Xiao Li, ZhongHua Qiao, and Hui Zhang, An unconditionally energy stable finite difference scheme for a stochastic cahn-hilliard equation, Science China Mathematics, 59 (2016), pp. 1815-1834.

[33] Zhinui Liu and Zhonghua Qiao, Strong approximation of monotone stochastic partial different equations driven by multiplicative noise, arXiv preprint arXiv:1811.05392, (2018).

[34] - Strong approximation of monotone stochastic partial differential equations driven by white noise, IMA J. Numer. Anal., (2019).

[35] Gabriel J Lord, Catherine E Powell, and Tony Shardlow, An Introduction to Computational Stochastic $P D E s$, no. 50, Cambridge University Press, 2014.

[36] A.K. Majee And A. Prohl, Optimal strong rates of convergence for a space-time discretization of the stochastic Allen-Cahn equation with multiplicative noise, Comput. Methods Appl. Math., 18 (2018), pp. 297-311.

[37] Ruisheng Qi and Xiaojie Wang, Optimal error estimates of Galerkin finite element methods for stochastic Allen-Cahn equation with additive noise, Journal of Scientific Computing, 80 (2019), pp. 1171-1194.

[38] Vidar Thomée, Galerkin finite element methods for parabolic problems, Springer-Verlag, Berlin, 2006. 